\title{
Methionine-Mediated Protein Phosphatase 2A Catalytic Subunit (PP2Ac) Methylation Ameliorates the Tauopathy Induced by Manganese in Cell and Animal Models
}

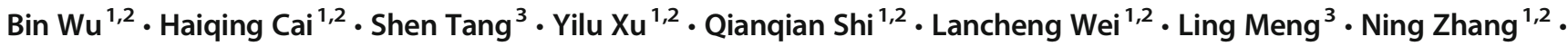 \\ Xinhang Wang ${ }^{3}$. Deqiang Xiao ${ }^{1,2} \cdot$ Yunfeng Zou ${ }^{1,2} \cdot$ Xiaobo Yang ${ }^{1,2} \cdot$ Xiyi Li ${ }^{1,2} \cdot$ Cailing Lu $^{1,2}$
}

Accepted: 4 September 2020 / Published online: 21 September 2020

(C) The American Society for Experimental NeuroTherapeutics, Inc. 2020

\begin{abstract}
The molecular mechanism of Alzheimer-like cognitive impairment induced by manganese (Mn) exposure has not yet been fully clarified, and there are currently no effective interventions to treat neurodegenerative lesions related to manganism. Protein phosphatase 2 A (PP2A) is a major tau phosphatase and was recently identified as a potential therapeutic target molecule for neurodegenerative diseases; its activity is directed by the methylation status of the catalytic $\mathrm{C}$ subunit. Methionine is an essential amino acid, and its downstream metabolite S-adenosylmethionine (SAM) participates in transmethylation pathways as a methyl donor. In this study, the neurotoxic mechanism of Mn and the protective effect of methionine were evaluated in Mn-exposed cell and rat models. We show that Mn-induced neurotoxicity is characterized by PP2Ac demethylation accompanied by abnormally decreased LCMT-1 and increased PME-1, which are associated with tau hyperphosphorylation and spatial learning and memory deficits, and that the poor availability of SAM in the hippocampus is likely to determine the loss of PP2Ac methylation. Importantly, maintenance of local SAM levels through continuous supplementation with exogenous methionine, or through specific inhibition of PP2Ac demethylation by ABL127 administration in vitro, can effectively prevent tau hyperphosphorylation to reduce cellular oxidative stress, apoptosis, damage to cell viability, and rat memory deficits in cell or animal Mn exposure models. In conclusion, our data suggest that SAM and PP2Ac methylation may be novel targets for the treatment of Mn poisoning and neurotoxic mechanism-related tauopathies.
\end{abstract}

Key Words Methionine $\cdot$ ABL127 $\cdot$ manganism $\cdot$ tau $\cdot$ neurodegeneration $\cdot$ PP2Ac methylation

Bin Wu, Haiqing Cai and Shen Tang contributed equally to this work.

Xiyi Li

xiyi.li2017@hotmail.com

Cailing Lu

lucailing78@gxmu.edu.cn

1 School of Public Health, Guangxi Medical University, 22 Shuangyong Road, Nanning 530021, Guangxi, People's Republic of China

2 Guangxi Colleges and Universities Key Laboratory of Prevention and Control of Highly Prevalent Diseases, Guangxi Medical University, 22 Shuangyong Road, Nanning 530021, Guangxi, People's Republic of China

3 School of Basic Medical Sciences, Guangxi Medical University, 22 Shuangyong Road, Nanning 530021, Guangxi, People's Republic of China

\section{Introduction}

Although manganese $(\mathrm{Mn})$ is an essential trace metal to maintain the homeostasis of the nervous system in mammalian animals [1,2], overexposure to Mn from occupational settings and the ambient environment leads to neurodegeneration characterized by progressive motor dysfunction [3, 4]. In addition, $\mathrm{Mn}^{2+}$ can be used as a contrast agent for Mn-enhanced magnetic resonance imaging (MEMRI) [5]. Recent studies have shown that in MEMRI, focal Mn injection, or systemic injection may have toxic effects on neurons $[6,7]$.

The full range of causative factors in manganism remains elusive despite a long period of study, and there are currently no effective interventions to treat neurodegenerative lesions related to Mn exposure. Recent investigations have indicated that elevated $\mathrm{Mn}$ exposure is likely a risk factor for Alzheimer's disease (AD) $[8,9]$ since patients with elevated Mn levels exhibit typical AD dementia symptoms as well as 
pathological signs of nerve plaques and neurofibrillary tangles (NFTs). Epidemiological studies have disclosed that chronic Mn exposure through drinking water causes cognitive impairment in children [10]. Animal models also suggested that $\mathrm{Mn}$ treatments led to decreased cognitive function, including impaired spatial working memory [11]. Due to the unique ADlike lesions, the mechanism of manganism has been widely studied by scholars.

Tau, as a highly water-soluble phosphorylated protein, is enriched in the axons of mature neurons to maintain microtubule stability and nerve function, and its phosphorylation is regulated by the balance between the activity of protein kinases and the activity of phosphoprotein phosphatases [12-15]. In recent years, a variety of neurotoxicants, such as mercury, lead, and 1-methyl-4phenyl-1,2,3,6-tetrahydropyridine (MPTP), have been found to be closely linked to tau hyperphosphorylation [16-18]. Multiple protein kinases were reported to be involved in tau hyperphosphorylation induced by Mn exposure in vitro [19]. Hyperphosphorylated tau is vulnerable to disconnection from microtubules and abnormally aggregates to form NFTs, which is one of the classic pathologies of AD [20, 21]. Moreover, hyperphosphorylated tau tangles and the accumulation of tau can cause excessive production of reactive oxygen species (ROS). The release of ROS can cause neuron damage and even neuronal apoptosis, which eventually manifest as cognitive impairment [22]. Therefore, the prevention of tau hyperphosphorylation to avoid neuronal cell damage may be a crucial event for the treatment of related neurodegenerative diseases.

Compared to the large number of protein kinases, a smaller number of protein phosphatases represented by protein phosphatase 1 (PP1) and protein phosphatase 2A (PP2A) are responsible for the specific dephosphorylation of thousands of phosphoprotein substrates [23]. PP2A is a ubiquitously expressed serine/threonine phosphatase, and its heterotrimeric holoenzymes consist of scaffolding subunit A, regulatory subunit $\mathrm{B}$, and highly conserved catalytic subunit $\mathrm{C}$. The interaction of the $\mathrm{C}$ subunit and the $\mathrm{B}$ subunit determines substrate specificity and regulation. The activation of PP2A involves complex processes, including protein-protein interactions and posttranslational modification [24, 25]. The methylation of the $\mathrm{C}$ subunit regulates PP2A phosphatase activity by controlling the binding of regulatory $\mathrm{B}$ subunits to AC dimers, while the demethylation of the $\mathrm{C}$ subunit is closely related to PP2A inactivation [26]. The PP2Ac-specific leucine carboxyl methyltransferase LCMT-1 and the specific methylesterase PME-1 participate in the regulation of PP2Ac methylation at the leu309 residue [27]. Studies have indicated that hypomethylated PP2A along with LCMT-1 reduction and PME-1 promotion is responsible for tauopathies in $\mathrm{AD}$ patients [28-31].
Methionine, as essential amino acid, and its downstream metabolite S-adenosylmethionine (SAM) donate methyl groups to a large variety of acceptor molecules, including DNA, histones, and proteins, to maintain the normal physiological processes of the nervous system [32-34]. Notably, some evidence from animal and clinical trials has shown that excessive methionine intake likely contributes to AD-like neurodegeneration [35]. In addition, early studies found that methionine levels decreased in a particular area of the brain of mice treated with $\mathrm{Mn}$ [36]. Therefore, the role of methionine in $\mathrm{Mn}$ poisoning and AD-related neuropathy is worthy of indepth study.

Given the above, we decided to investigate the pathogenesis of $\mathrm{Mn}$ poisoning and the role of the methionine/SAMreactivated PP2Ac methylation pathway in Mn neurotoxicity. As a result, we found that Mn exposure promotes the hypomethylation of PP2Ac by reducing the availability of SAM in $\mathrm{N} 2 \mathrm{a}$ cells and the hippocampus of rats, leading to the hyperphosphorylation of tau protein. Importantly, recovering PP2Ac methylation through continuous administration of methionine could effectively prevent the Mn-induced full spectrum of AD-like tauopathy characteristics, including memory deficits. Moreover, ABL127, a PP2A activator that specifically inhibits PME-1 [37], effectively relieved tau hyperphosphorylation, oxidative stress, apoptosis, and damage to cell viability in Mn-exposed N2a cells by recovering PP2Ac methylation.

\section{Material and Methods}

\section{Chemicals}

Methionine-free medium (RPMI-1640 medium, R7513), methionine powder (L-methionine, M9625, purity > 98\%), manganese chloride ( $\mathrm{MnCl} 2 \cdot 4 \mathrm{H} 2 \mathrm{O}, \mathrm{M} 3634$, purity $>99 \%)$, and ABL127 (SML0294, purity > 98\%) were purchased from Sigma Chemical Co. (St. Louis, MO). Demethylated PP2Ac, methylated PP2Ac, LCMT-1, PME1, and total PP2Ac antibodies were obtained from Santa Cruz Biotechnology (Santa Cruz, CA). Phosphorylated (P)-tau (Ser199, Ser396, Ser404) and total tau (tau-5) antibodies were purchased from Abcam Co. Cyclophilin B was purchased from Cell Signaling Technology Co. (Danvers, MA).

\section{Cell Culture and Treatment}

N2a cells, a mouse neuroblastoma cell line, were obtained from Shanghai Institutes for Biological Sciences (Shanghai, China) and cultured in minimum essential medium (MEM) containing $10 \%$ fetal bovine serum and $1 \%$ antibiotics (100 units $/ \mathrm{mL}$ ) in a $5 \% \mathrm{CO}_{2}$ incubator at $37{ }^{\circ} \mathrm{C}$. At $80 \%$ confluence, the cells were plated into either a 6 -well plate or 
a 96-well plate containing methionine-free RPMI-1640 medium and exposed to $500 \mu \mathrm{M}$ and $1000 \mu \mathrm{M} \mathrm{MnCl}_{2} \cdot 4 \mathrm{H}_{2} \mathrm{O}$ with or without incubation with $10 \mathrm{mg} / \mathrm{L}$ methionine or $0.5 \mathrm{nM}$ ABL127. The morphology of N2a cells from each treatment group was observed by an inverted microscope (DMi8; Leica, Germany). After $24 \mathrm{~h}$ of treatment, the cells were collected for subsequent analyses.

\section{Cell Viability Determined by alamarBlue Assay}

N2a cells were seeded in 96-well plates at a density of $2 \times$ $10^{4}$ cells/well. After $24 \mathrm{~h}$ of treatment, $100 \mu \mathrm{L}$ of $1 \%$ alamarBlue Cell Viability Reagent (Thermo Fisher, USA) was added to each well and incubated again at $37^{\circ} \mathrm{C}$ for $4 \mathrm{~h}$. Finally, the optical density of cells was detected at wavelengths of $570 \mathrm{~nm}$ and $600 \mathrm{~nm}$ with a plate reader (Protein Simple, USA).

\section{Detection of Apoptosis by Flow Cytometry}

N2a cell apoptosis was quantified by flow cytometry. The N2a cells were washed three times with prechilled PBS at $4{ }^{\circ} \mathrm{C}$ for $3 \mathrm{~min}$. The cells were digested with trypsin without EDTA for $3 \mathrm{~min}$ at $37^{\circ} \mathrm{C}$. The cells in suspension were then centrifuged at $1000 \mathrm{rcf}$ for $5 \mathrm{~min}$. The supernatant was discarded, and the cells were washed with PBS and then centrifuged at $1000 \mathrm{rcf}$ for $5 \mathrm{~min}$. Next, we resuspended the pellet in $100 \mu \mathrm{L}$ of binding buffer, $5 \mu \mathrm{L}$ of Annexin V-FITC, and $5 \mu \mathrm{L}$ of propidium iodide. The sample was incubated for $15 \mathrm{~min}$ at room temperature in the dark, and $400 \mu \mathrm{L}$ of loading buffer was added. The samples were detected by flow cytometry (CytoFLEX; Beckman Coulter, USA) within $1 \mathrm{~h}$. Each sample was analyzed in triplicate.

\section{Determination of Oxidative Stress Level}

The level of ROS in cells induced by the above treatment after $24 \mathrm{~h}$ was tested by a 2,7-dichlorofluorescein diacetate (DCFHDA) assay (Beyotime, China). Briefly, DCFH-DA $(10 \mu \mathrm{mol} / \mathrm{L})$ was added, and the cells were incubated at $37^{\circ} \mathrm{C}$ for $30 \mathrm{~min}$ and then washed twice with PBS. Subsequently, the fluorescence intensity was read by a multifunction microplate reader (excitation wavelength of $488 \mathrm{~nm}$ and emission wavelength of $525 \mathrm{~nm}$ ). The GSH content of cells was detected by the GSH and GSSG Assay Kit (Beyotime, China), and the specific method was based on previous research [38]. Briefly, total glutathione (GSSG plus GSH) was determined spectrophotometrically with DTNB and NADPH at $412 \mathrm{~nm}$. GSSG was measured in the same way in the presence of 2-vinylpyridine, and the amount of GSH was calculated by subtracting the amount of GSSG from the amount of total glutathione.
Animals, Treatments, and Sample Collection

All animal studies were approved by the ethical committee for animal experiments of Guangxi Medical University (GXMU2014-036) and performed in accordance with the international standards of the Guidelines for the Care and Use of Laboratory Animals in animal experiments. This study strived to minimize the number and suffering of experimental animals. Male Sprague-Dawley rats $(200 \pm 10 \mathrm{~g}$ weight, SPF grade) were provided by the experimental animal center of Guangxi Medical University (LicenseNo:SCXKGui20090002) and were kept on a 12-h light-dark cycle at a room temperature of $21-23{ }^{\circ} \mathrm{C}$ with free access to food and water. After a week of adaptive feeding, 80 rats were randomly assigned to four 4-week and four 16-week exposure groups (10 rats/group), namely, the control group, Mn model group, methionine control group, and methionine plus Mn group. $\mathrm{Mn}$-administered rats were injected intraperitoneally with $\mathrm{MnCl}_{2} \cdot 4 \mathrm{H}_{2} \mathrm{O}$ (15 mg/kg, in saline). In the methionine control group, methionine $(200 \mathrm{mg} / \mathrm{kg}$, in saline) was administered. The rats in the methionine plus Mn group were injected intraperitoneally with $15 \mathrm{mg} \mathrm{MnCl} \cdot 4 \mathrm{H}_{2} \mathrm{O} / \mathrm{kg}$ and $200 \mathrm{mg}$ methionine $/ \mathrm{kg}$. Control animals received saline $(0.9 \% \mathrm{NaCl})$ injection. The injection was given every day. The volume of administration was $5 \mathrm{~mL} / \mathrm{kg}$. After behavioral testing, blood samples were collected from the abdominal aorta in heparintreated blood collection tubes. The brains were quickly removed and placed on a plastic petri dish with cold saline. The hippocampus and cortex from one half of the whole brain were dissected, and the other half was spared. All samples were stored at $-80^{\circ} \mathrm{C}$.

\section{Morris Water Maze Test}

The Morris water maze (MWM) test was used to determine changes in spatial learning and memory ability $24 \mathrm{~h}$ after the last injection. The experimental device was a cylindrical pool, $160 \mathrm{~cm}$ in diameter and $60 \mathrm{~cm}$ in height, which was divided into four quadrants: quadrants I, II, III, and IV. A black circular platform with a diameter of $12 \mathrm{~cm}$ was placed in quadrant III and was $2 \mathrm{~cm}$ below the surface of the water. The objects around the water maze were kept fixed, and the water temperature was stable at $23 \pm 2{ }^{\circ} \mathrm{C}$. A camera was suspended $2 \mathrm{~m}$ above the maze, and a computer equipped with Morris water maze analysis software (Huaibei Zhenghua Biological Equipment Co., China) was used to record the swimming track in the water maze. All tests were started at 8:00 am on 6 consecutive days. In the first 5 days, each rat entered the maze from four different quadrants and trained four times a day, with a training interval of $30 \mathrm{~s}$. When a rat did not find the platform within $60 \mathrm{~s}$, the tester guided it to the platform and recorded an escape latency of $60 \mathrm{~s}$. On the 6th day, the platform was removed, and each rat was put into the maze in a 
fixed quadrant. The time (s) spent in the target quadrant and the probe time were used to evaluate the level of animal spatial memory.

\section{Analysis of Manganese Content in the Blood and Brain of Rats}

At the end of the behavioral study, whole blood and brain samples were collected from rats in each group after decapitation. The samples were prepared by digestion with nitric acid $(65 \%)$ in a microwave digestion apparatus (MARS6; CEM, Matthews, NC, USA). The level of Mn was determined by inductively coupled plasma mass spectrometry (ICP-MS, X Series 2; Thermo Scientific, Waltham, MA, USA) (6 samples per treatment group).

\section{Western Blot Analysis}

Whole protein was extracted from cells by a 10-min incubation with RIPA medium lysis buffer containing protease inhibitor (P0013C, Beyotime, China). The rat hippocampi and cortexes were homogenized at $4{ }^{\circ} \mathrm{C}$ using a buffer containing RIPA strong lysate buffer (P0013B, Beyotime, China) and $1 \mathrm{mM}$ PMSF, and the supernatants were collected after centrifugation of the tissue homogenates at $12,000 \mathrm{rpm} / \mathrm{min}$ for $30 \mathrm{~min}$ at $4{ }^{\circ} \mathrm{C}$. The protein concentration was detected by the Bicinchoninic Acid (BCA) Protein Assay Kit (TaKaRa, Japan) according to the vendor's protocol. Samples containing the same amount of protein were loaded onto an SDSpolyacrylamide gel. After separation, the proteins were transferred to a PVDF membrane followed by blocking of nonspecific binding sites and treatment with antibodies. Membranes were incubated overnight at $4{ }^{\circ} \mathrm{C}$ with the primary antibodies and then washed 3 times with TBST and incubated with the secondary antibody, goat anti-rabbit $\operatorname{IgG}(1: 2000)$ or antimouse $\operatorname{IgG}(1: 2000)$, for $2 \mathrm{~h}$. The profile of primary and secondary antibodies tested in this study is shown in Table 1.
Protein expression was detected by a chemiluminescence assay and quantitated by an imaging analysis system (FL1000, Thermo Fisher, USA). Standardization was performed with cyclophilin B (CYPB).

\section{Quantification of SAM and SAH in Brain Tissue and Cells}

SAM and SAH were determined by ultrahigh-performance liquid chromatography-tandem mass spectrometry (UPLCMS). SAM and SAH were extracted from brain tissues and cultured cells. Briefly, counted cells were collected in $1.5-\mathrm{mL}$ Eppendorf tubes containing $200 \mu \mathrm{L}$ of the initial mobile phase $(5.0 \mathrm{mmol} / \mathrm{L}$ ammonium acetate $+0.4 \%$ acetic acid $+2 \%$ methanol) and repeatedly frozen and thawed at $-80^{\circ} \mathrm{C}$ three times. Tissues frozen at $-80{ }^{\circ} \mathrm{C}$ were removed and placed in 1.5-mL Eppendorf tubes containing $200 \mu \mathrm{L}$ of extraction buffer, and the tissues were fully ground with a handheld homogenizer, followed by sonication at $40 \mathrm{~W}$ and $60 \%$ duty cycle for approximately $40 \mathrm{~s}$. Then, all samples were clarified by centrifugation at $10,000 \times g$ for $10 \mathrm{~min}$ at $4{ }^{\circ} \mathrm{C}$. The supernatant was taken and brought to volume at $1 \mathrm{~mL}$, passed through an organic phase filter $(0.22 \mu \mathrm{m}, 13 \mathrm{~mm})$, and tested within $24 \mathrm{~h}$ after bottling. The levels of SAM and SAH were assayed using a UPLC system (CORTES, Waters Corp, USA) interfaced with a mass spectrometer (QTRAP 4500, AB Sciex, USA). Briefly, the samples were separated on a CORTES UPLC-C18+ column and subjected to multiple reaction monitoring (MRM) scanning in electrospray ion source positive ion (ESI+) mode. The column temperature was maintained at $40{ }^{\circ} \mathrm{C}$, the flow rate was $0.35 \mathrm{~mL} / \mathrm{min}$, and the gradient elution process was as follows: buffer A: $0.4 \%(\mathrm{v} / \mathrm{v})$ acetic acid aqueous solution containing $10 \mathrm{mmol} / \mathrm{L}$ ammonium acetate; buffer B: methanol. $T=0 \mathrm{~min}, 0 \% \mathrm{~B} ; T=1 \mathrm{~min}$, $2 \% \mathrm{~B} ; T=3 \mathrm{~min}, 75 \% \mathrm{~B} ; T=4 \mathrm{~min}, 2 \% \mathrm{~B}$; and $T=5 \min , 2 \%$ B. The ESI source voltages were $5.5 \mathrm{kV}$ with a capillary temperature of $400{ }^{\circ} \mathrm{C}$; CUR: $206 \mathrm{kPa}$; collision gas (CAD):
Table 1 Antibodies used in the study

\begin{tabular}{lllll}
\hline Antibodies & Epitopes & Type & Dilution & Sources \\
\hline PP2Ac & PP2A-C subunit & $\mathrm{mAb}$ & $1 / 2000$ & Santa Cruz (CA, USA) \\
Dem-PP2Ac & Demethylated-PP2A-C submit & $\mathrm{mAb}$ & $1 / 2000$ & Santa Cruz (CA, USA) \\
Me-PP2Ac & Methyl-PP2A-C submit & $\mathrm{mAb}$ & $1 / 500$ & Santa Cruz (CA, USA) \\
LCMT-1 & LCMT-1 & $\mathrm{mAb}$ & $1 / 1000$ & Santa Cruz (CA, USA) \\
PME-1 & PME-1 & $\mathrm{mAb}$ & $1 / 1000$ & Santa Cruz (CA, USA) \\
pS396 & Phospho-tau at Ser 396 & $\mathrm{mAb}$ & $1 / 2000$ & Abcam (Cambridge, UK) \\
pS199 & Phospho-tau at Ser 199 & $\mathrm{mAb}$ & $1 / 2000$ & Abcam (Cambridge, UK) \\
pS404 & Phospho-tau at Ser 404 & $\mathrm{mAb}$ & $1 / 2000$ & Abcam (Cambridge, UK) \\
Tau-5 & Tau-5 (total tau) & $\mathrm{mAb}$ & $1 / 2000$ & Abcam (Cambridge, UK) \\
CYPB & Cyclophilin B & $\mathrm{mAb}$ & $1 / 2000$ & Beyotime (Shanghai, China) \\
\hline
\end{tabular}

$m A b$ monoclonal antibody 
medium; GS1: $384 \mathrm{kPa}$; and GS2: $384 \mathrm{kPa}$. The injection volume was $2 \mu \mathrm{L}$, and triplicate injections were performed for each sample. The area under each peak was quantified using software, and the accuracy was re-examined. The results were quantified by an external standardization method.

\section{Data Analysis}

All data are presented as means \pm SDs or means \pm SEMs. The statistical analyses were performed using SPSS 23.0 statistical software (SPSS Inc., Chicago, IL, USA). Statistical graphs were generated using GraphPad Prism 6 statistical software (GraphPad Software Inc., San Diego, CA, USA). Student's $t$ test was used for two-group comparisons, and one-way ANOVA was used to determine the differences among groups, followed by LSD's post hoc test to determine the differences between two groups. The difference was considered significant when $p<0.05$.

\section{Results}

\section{Exogenous Methionine Is Indispensable for N2a Cells to Resist Mn-Induced Tau Neurotoxicity}

\section{Methionine Deficiency Increases the Sensitivity of N2a Cells to Mn Injury}

We constructed a methionine-free medium suitable for cell growth to create a methionine-deficient condition for $\mathrm{N} 2 \mathrm{a}$ cells and took $10 \mathrm{mg} / \mathrm{L}$, the most suitable concentration for cell viability, as a supplemental concentration of exogenous methionine to ensure sufficient methionine intake (Fig. S1a, S1b). To preliminarily evaluate the adverse effects of $\mathrm{Mn}$ on the cell viability of methionine-deficient $\mathrm{N} 2 \mathrm{a}$ cells, N2a cells were treated with increasing concentrations of $\mathrm{Mn}(0-10,000 \mu \mathrm{M})$ for $24 \mathrm{~h}$ in methionine-free medium. Cell viability analysis after $24 \mathrm{~h}$ of incubation revealed an $\mathrm{LC}_{50}$ of $1600 \mu \mathrm{M}$. When the Mn concentration was greater than or equal to $500 \mu \mathrm{M}$, the cell viability was decreased in a concentration-dependent fashion. At concentrations higher than $1000 \mu \mathrm{M}$, the survival rate of cells was less than $65 \%$. These two sublethal concentrations of $\mathrm{Mn}$ were selected for subsequent experiments (Fig. S1c, S1d).

The N2a cells were treated with methionine or Mn individually or jointly for $24 \mathrm{~h}$ in the next experiments. As the $\mathrm{Mn}$ concentration increased, we observed that the N2a cells appeared round, and the bulge on the cell surface disappeared (Fig. 1a). However, the restoration of the supply of exogenous methionine significantly improved the cell viability of $\mathrm{Mn}$ exposed N2a cells (Fig. 1b). Furthermore, the results of apoptosis detection showed that cells subjected to Mn exposure at
$500 \mu \mathrm{M}$ and $1000 \mu \mathrm{M}$ had more evident apoptosis, with rates of $14.08 \%$ and $22.45 \%$, respectively, than the untreated cells in methionine-free culture, while sufficient methionine resulted in a significant reduction in Mn-induced apoptosis (Fig. $1 \mathrm{c}, \mathrm{d})$.

Studies have shown that excessive ROS may be a direct cause of neuronal damage and apoptosis [39]. To determine whether added methionine modulated cellular redox homeostasis under $\mathrm{Mn}$ exposure, the levels of ROS and GSH were detected. Our results showed that the levels of ROS in cells increased markedly upon $\mathrm{Mn}$ treatment, while methionine supplementation significantly reduced ROS levels in Mn-exposed N2a cells (Fig. le, f). In addition, $\mathrm{Mn}$ exposure reduced the ratio of GSH to GSSG in methionine-free culture, and methionine deficiency independently reduced the ratio of GSH to GSSG. As expected, the ratio of GSH to GSSG in Mn-exposed cells was significantly increased after methionine treatment (Fig. 1g). These findings indicated that N2a cells may rely on exogenous methionine to maintain redox homeostasis maximally to resist $\mathrm{Mn}$-induced cell damage.

\section{Mn-Exposed N2a Cells Depend on Sufficient Methionine to Avoid Abnormal Phosphorylation of Tau by Maintaining PP2Ac Hypermethylation}

The hyperphosphorylation of tau and the accumulation of phosphorylated tau can stimulate ROS production, causing oxidative stress and neuronal cell damage [40]. In this experiment, we first examined the tau phosphorylation levels at AD-relevant sites by Western blot (Fig. 2a). The results showed that $\mathrm{Mn}$ increased the levels of tau phosphorylation at Ser199, Ser396, and Ser404 in a concentration-dependent fashion in methionine-deficient groups. However, when exogenous methionine was sufficient, Mn-induced tau hyperphosphorylation at specific sites was completely suppressed (Fig. 2b, c, e).

Tau dephosphorylation is directly regulated by activated PP2A, and the activity of PP2A is inhibited partially through the loss of catalytic subunit methylation at Leu309 [41]. We next detected methylated and demethylated PP2A protein expression (Fig. 2d). With increasing Mn exposure concentration, the PP2Ac methylation level of methionine-deficient cells showed a downward trend (Fig. 2g), whereas the level of demethylation was increased (Fig. 2h). However, the abovementioned Mn-induced alteration in the PP2Ac methylation pattern was avoided in the presence of sufficient exogenous methionine (Fig. 2g-i). These data suggest that Mnexposed N2a cells require exogenous methionine to resist the tau hyperphosphorylation caused by continuously downregulated levels of PP2Ac methylation. 
a

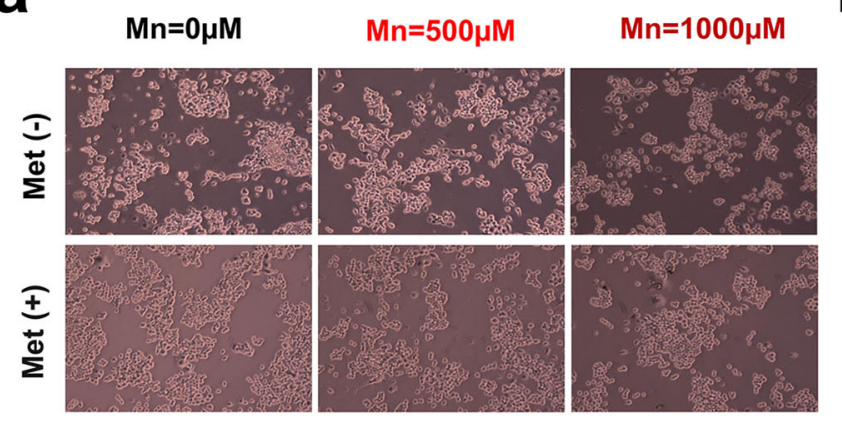

C
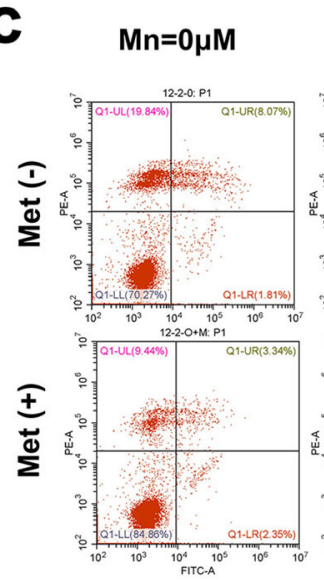

e

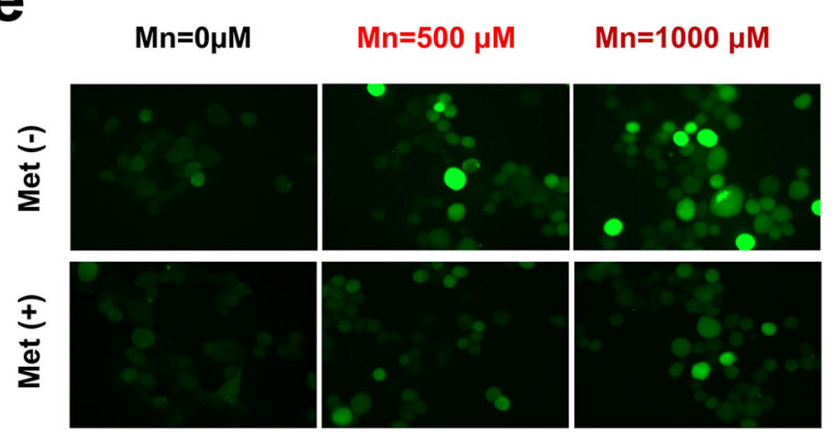

Fig. 1 N2a cells depended on methionine to resist Mn toxicity. Morphology images in Mn-exposed N2a cells incubated with or without methionine (a). Alamar blue assay for cell viability in Mnexposed N2a cells incubated with or without methionine (b). Flow cytometry for detection of apoptosis level in Mn-exposed N2a cells incubated with or without methionine (c, d). Fluorescence image of ROS in Mn-exposed N2a cells incubated with or without methionine and quantification of ROS levels $(\mathbf{e}, \mathbf{f})$. The ratio of GSH to GSSG in

\section{SAM Levels Affect the Balance of LCMT-1 and PME-1 Expression in Mn-Exposed N2a Cells}

LCMT-1 transports the methyl group for PP2Ac at the Leu309 site, PME-1 directly binds to the activation site to remove the methyl group, and the two work together to maintain the PP2Ac methylation status and transmethylation function [42, 43]. Recent studies have shown that SAM levels can regulate b
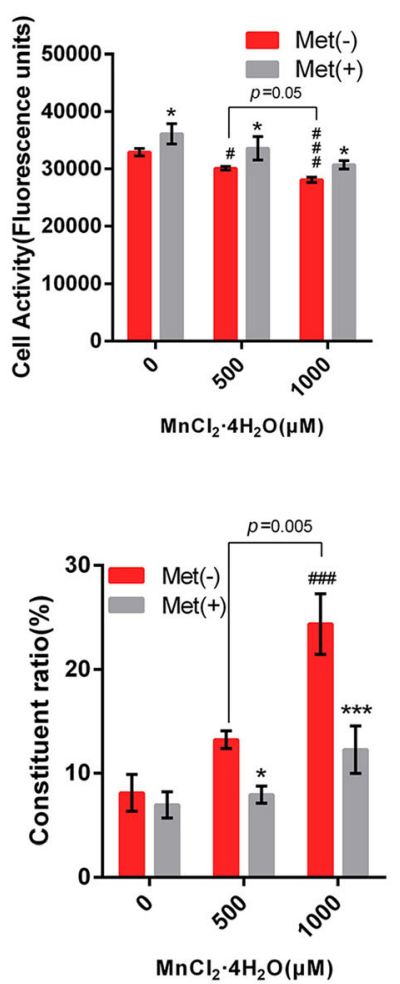

f

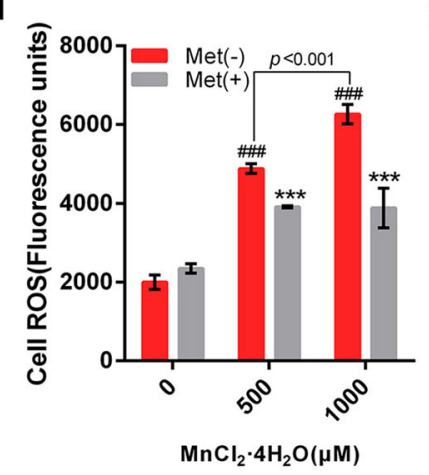

g

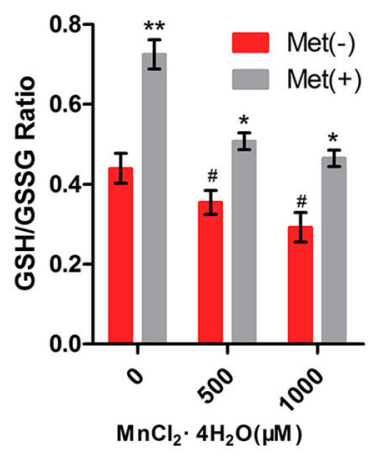

Mn-exposed N2a cells incubated with or without methionine (g). The data shown represent the mean $\pm \mathrm{SD}, n=3$. Student's $t$ test, $* p<0.05$ and $* * * p<0.005$ versus the Met $(-)$ group at the equal Mn treatment concentration. One-way ANOVA (effect of $\mathrm{Mn}$ in methionine deprivation), in (b), for cell viability, $F(2,6)=73.202, p<0.001$; in (d), for apoptosis, $F(2,6)=50.590, p<0.001$; in (f), for ROS, $F(2,6)=$ $383.989, p<0.001$; and in $(\mathbf{g})$, for $\mathrm{GSH} / \mathrm{GSSG}, F(2,6)=5.345, p=$ $0.046 ;{ }^{\#} p<0.05$ and ${ }^{\# \# \#} p<0.005$ versus Met $(-)$ control group

the methylation patterns of multiple methyl receptors in several systems, and reductions in methyltransferase activity and expression may be related to a low ratio of SAM to SAH [44, 45]. We hypothesized that $\mathrm{Mn}$ or methionine might regulate PP2Ac methylation status by affecting these upstream regulators. As shown in the results of the immunoblotting test (Fig. 3a), we observed reduced LCMT-1 (Fig. 3b) and elevated PME-1 (Fig. 3c) expression in methionine-deficient cells 
a

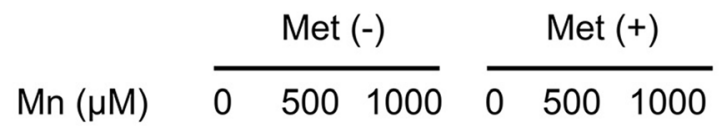

pS199

pS396

pS404

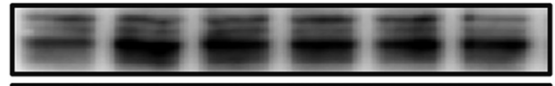

Tau-5

CYPB

d

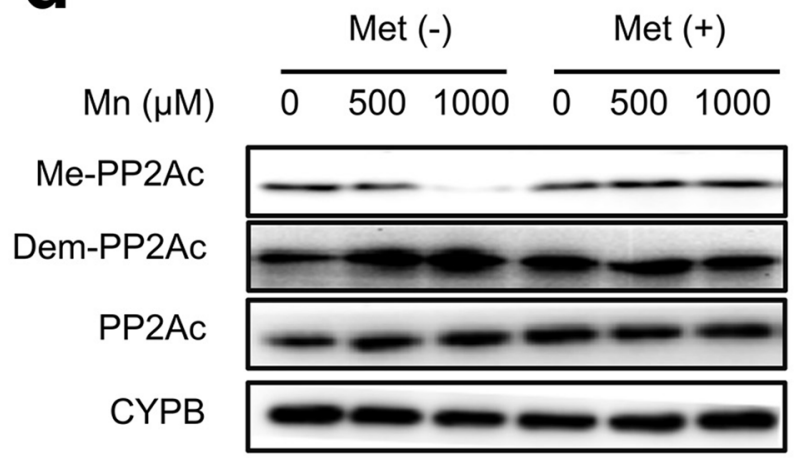

g

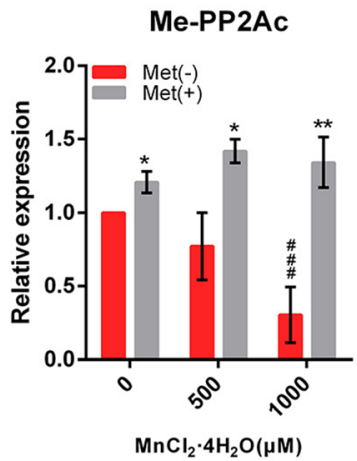

Fig. 2 Sufficient methionine incubation attenuated tau hyperphosphorylation by preventing loss of PP2Ac methylation in Mninduced N2a cells. Cellular protein levels of total tau and p-tau at Ser199, Ser396, and Ser404 were measured by WB (a-c, e, f). Cellular protein levels of total PP2Ac, methylated PP2Ac, and demethylated PP2Ac were measured by WB $(\mathbf{d}, \mathbf{g}-\mathbf{i})$. All data are presented as mean \pm SD. Student's $t$ test, $* p<0.05$ and $* * * p<0.005$ versus the Met $(-)$ group at the equal

upon $\mathrm{Mn}$ exposure. Methionine deficiency did not exert an independent effect on the expression of LCMT-1 or PME-1. However, sufficient methionine allowed Mn-exposed cells to have near-normal levels of LCMT-1 expression while exhibiting lower PME-1 levels than methionine deficiency (Fig. 3a-c). We next measured the intracellular levels of SAM and SAH. In methionine-free culture, Mn-treated cells b

Ser199

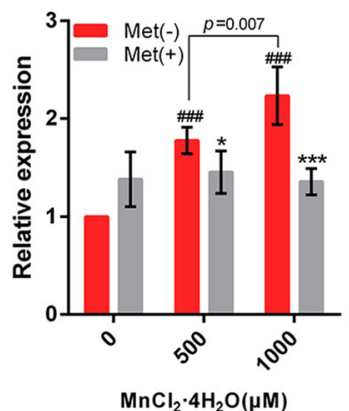

e

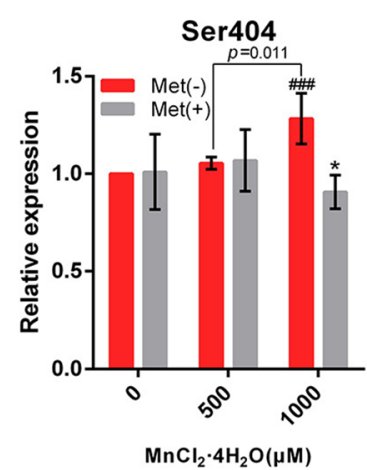

Tau-5

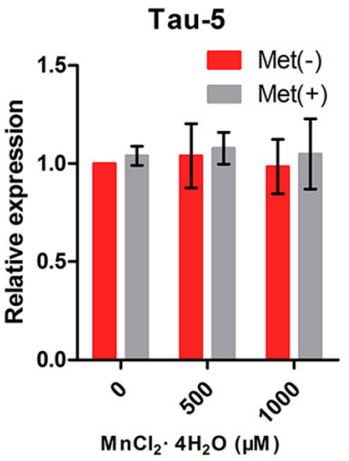

h
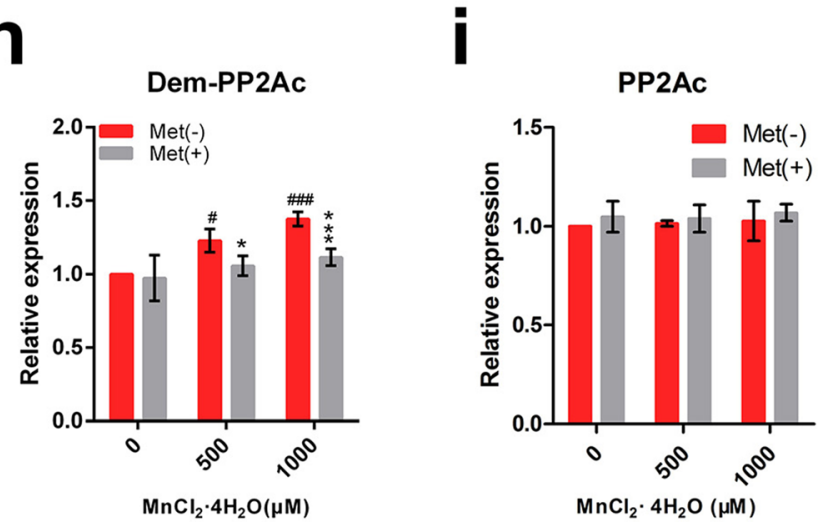

Mn treatment concentration. One-way ANOVA (effect of $\mathrm{Mn}$ in methionine deprivation), in (b), for Ser199, $F(2,9)=44.513, p<0.001$ $(n=4)$; in $(\mathbf{c})$, for Ser396, $F(2,9)=6.768, p=0.016(n=4)$; in $(\mathbf{e})$, for Ser404, $F(2,9)=11.296, p=0.009(n=4)$; in $(\mathbf{g})$, for me-PP2Ac, $F(2$, $6)=12.788, p=0.007(n=3)$; and in (h), for dem-PP2Ac, $F(2,6)=$ 29.557, $p=0.001(n=3) .{ }^{\#} p<0.05,{ }^{\# \#} p<0.01$, and ${ }^{\# \#} p<0.005$ versus Met (-) control group

showed reduced SAM levels and reduced ratios of SAM to SAH (Fig. 3d). However, the addition of methionine relieved the restricted SAM levels and increased the ratio of SAM to $\mathrm{SAH}$ in N2a cells treated with $1000 \mu \mathrm{M}$ Mn (Fig. 3e). These observations suggested that alterations in LCMT-1, PME-1, and SAM levels were involved in Mn-induced PP2A inactivation. Exogenous methionine seemed to act as a SAM sink to 


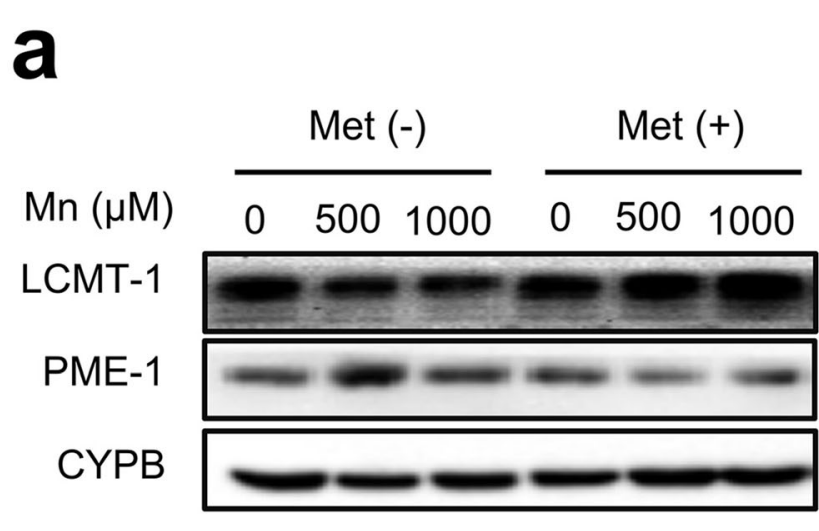

b C

LCMT1

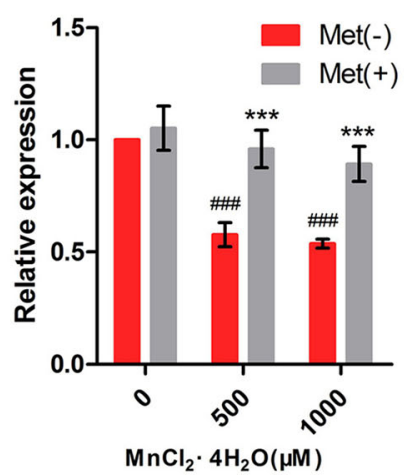

PME1

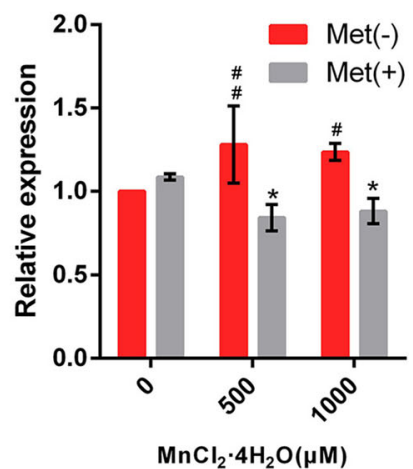

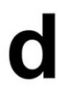

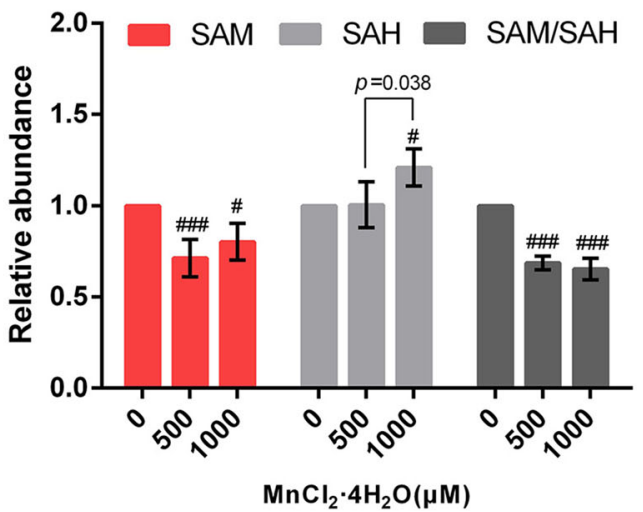

Fig. 3 Sufficient methionine incubation prevented imbalance between LCMT-1 and PME-1 by restoring SAM levels in Mn-exposed N2a cells. Cellular protein levels of LCMT-1 and PME-1 were measured by WB $(\mathbf{a}-\mathbf{c})$. Mn exposure reduced SAM levels and the ratio of SAM to $\mathrm{SAH}$ in N2a cells incubated without methionine (d). Addition of methionine increased SAM levels and the ratio of SAM to SAH in Mnexposed N2a cells (e). All data are expressed as means $\pm \mathrm{SD}, n=3$.

maintain SAM levels and avoid the abnormal expression of LCMT-1 and PME-1 under Mn exposure.

\section{Methionine Intervention Prevents Cognitive Impairment Caused by In Vivo Mn Exposure}

\section{The Role of Methionine in Spatial Learning and Memory and $\mathrm{Mn}$ Accumulation in Mn-Exposed Rats}

Based on the in vitro results, we found that Mn-treated N2a cells exhibited AD-like tau hyperphosphorylation, oxidative stress, apoptosis, and a high dependence on exogenous methionine. To explore the in vivo protective effect of methionine on Mn neurotoxicity, rat models of subacute (4week) and chronic (16-week) Mn exposure were established by intraperitoneal administration (Fig. 4a). It is well known that $\mathrm{AD}$ is a common brain disease associated with progressive memory loss and cognitive decline.

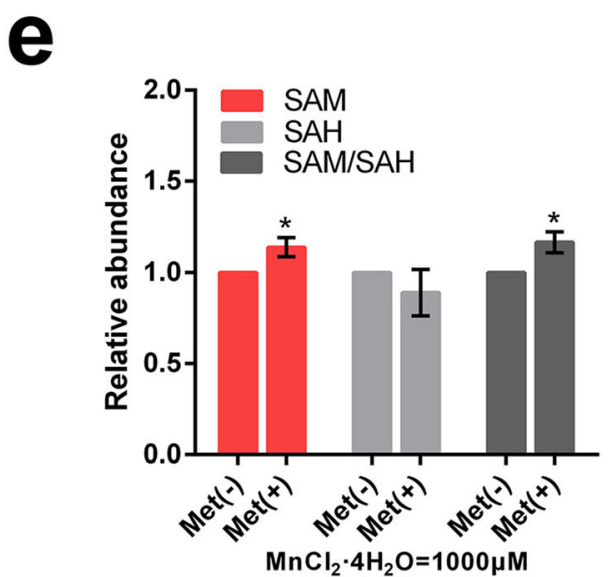

Student's $t$ test, $* p<0.05$ and $* * * p<0.005$ versus the Met (-) group at the equal $\mathrm{Mn}$ treatment concentration. One-way ANOVA (effect of Mn in methionine deprivation), in (b), for LCMT-1, $F(2,6)=59.380, p<0.001$; in (c), for PME-1, $F(2,6)=6.172, p=0.035$; in (d), for SAM, $F(2,6)=$ 9.405, $p=0.014$; for SAH, $F(2,6)=4.870, p=0.055$; for SAM $/ \mathrm{SAH}$, $F(2,6)=67.644, p<0.001$. ${ }^{\#} p<0.05,{ }^{\# \#} p<0.01$, and ${ }^{\# \# \#} p<0.005$ versus Met (-) control group

Therefore, we evaluated the effect of methionine on spatial learning and memory in Mn-exposed rats by the Morris water maze behavioral test. In order to avoid differences in the age and weight of rats due to different treatment durations, we analyzed the results of 4 weeks and 16 weeks respectively. As a result, in the training period, both subacute and chronic Mn administration resulted in a longer latency on the 4th and 5th days than saline injection, and the differences were more significant in the 16-week experiment. However, treatment with methionine significantly reduced the escape latency of Mn-exposed rats (Fig. 4b). In the spatial probe trial (Fig. 4c-f), rats that received 16 weeks of Mn administration showed decreased probe times and time spent in the target quadrant compared with the control group with no significant changes in swimming speed (Fig. 4c-e). However, methionine intervention greatly prevented these poor conditions (Fig. 4d, e). The above data showed that $\mathrm{Mn}$ impaired spatial learning and 
a

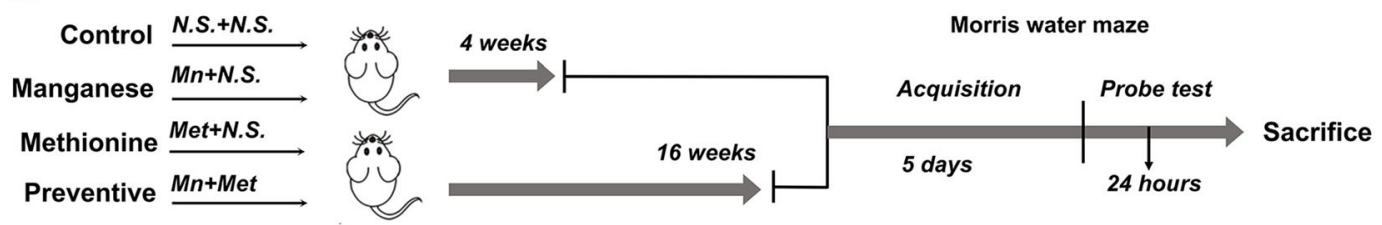

b
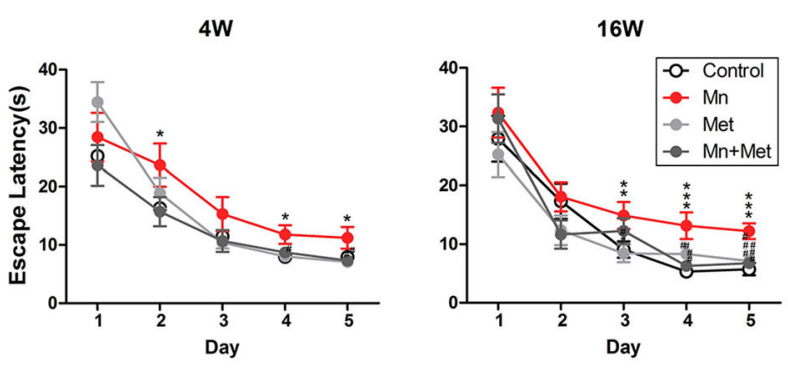

C

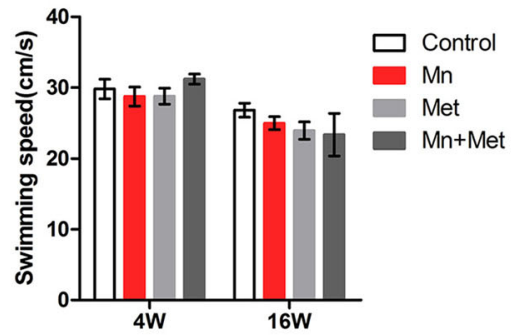

d
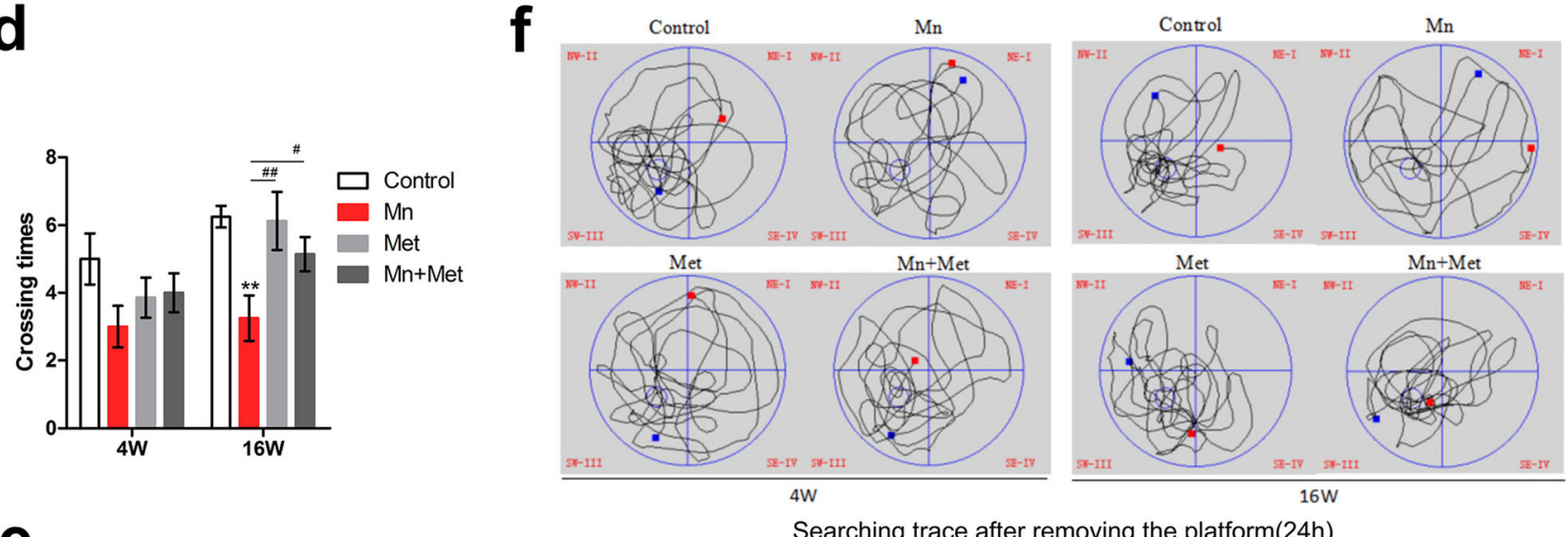

e

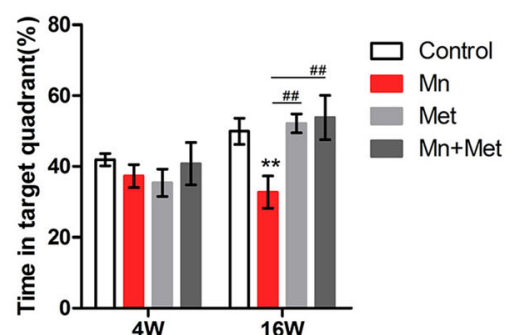

Fig. 4 Methionine prevents learning and memory deficits in Mn-exposed rats. The experimental design performed in the study (a). Escape latency in 5 days were analyzed in the MWM test (b). The swimming speed in the probe test (c). The times to cross the platform is shown (d). The percentage of time spent in target quadrant is shown (e). The searching trace in the probe test (f). The concentration of $\mathrm{Mn}$ in blood and hippocampus (g). In (c), for 4 weeks, $F(3,28)=0.441, p=0.726$; for 16 weeks, $F(3,28)=1.021, p=0.398$. In (d), for 4 weeks, $F(3,28)=$ $2.061, p=0.128$; for 16 weeks, $F(3,28)=5.202, p=0.006$. In $(\mathbf{e})$, for

memory in a time-dependent fashion and that methionine intervention effectively prevented this impairment.

We measured the Mn concentrations of whole blood and hippocampi by ICP-MS and found that the blood and hippocampal Mn levels were increased significantly in Mn-exposed
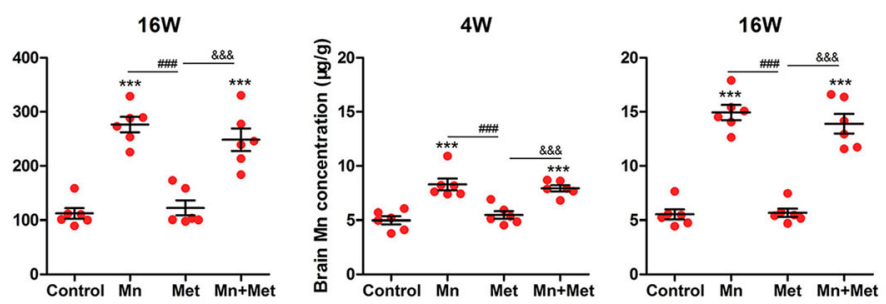

4 weeks, $F(3,28)=0.518, p=0.673$; for 16 weeks, $F(3,28)=5.341, p=$ 0.005. In (g), for blood, 4 weeks: $F(3,20)=10.221, p<0.001,16$ weeks: $F(3,20)=30.798, p<0.001$; for hippocampus, 4 weeks: $F(3,20)=$ 18.244, $p<0.001,16$ weeks: $F(3,20)=62.049, p<0.001$. One-way ANOVA, all data are expressed as means \pm SEM, $n=8(\mathbf{a}-\mathbf{f}), n=6(\mathbf{g})$. $* p<0.05, * * p<0.01$, and $* * * p<0.005$ versus the control group; ${ }^{\#} p<0.05$ and ${ }^{\# \#} p<0.01$ versus $\mathrm{Mn}$ group; ${ }^{\& \& \&} p<0.005$ versus methionine group

rats compared with those in the control group, and Mn accumulation increased with prolonged exposure. At the same time, the concentrations of $\mathrm{Mn}$ in rats treated with methionine showed a slight downward trend, but the difference was not significant (Fig. 4g). These data implied that Mn could 
selectively accumulate in the hippocampus of rats and that methionine did not attenuate Mn-induced cognitive dysfunction by reducing the accumulation of $\mathrm{Mn}$.

\section{Methionine Attenuates Tau Hyperphosphorylation by Inhibiting Excessive Demethylation of PP2AC in Mn-Exposed Rats}

Our in vivo results have confirmed that additional methionine intervention based on a normal diet significantly improves the cognition of Mn-exposed rats. Tau hyperphosphorylation is involved in learning and memory loss, and its dephosphorylation is regulated by PP2A [46]. Therefore, we measured the p-tau levels in the cortex and hippocampus of rats (Fig. 5a, b). As shown in the results, we observed a marked increase in tau phosphorylation at Ser396 in a time-dependent fashion in the cortex and hippocampus of Mn-exposed rats (Fig. 5c, d). Interestingly, the tau phosphorylation level at Ser404 in the Mn group was significantly increased compared to the level in the control group only in the 16-week experiment, but there was no change at Ser199 (Fig. S2). However, methionine intervention almost completely prevented the Mn-induced hyperphosphorylation of tau at Ser396 and Ser404 (Fig. 5c, d).

Furthermore, to confirm that methionine prevents tau hyperphosphorylation by activating PP2A in our Mnexposed rat model, we detected the PP2Ac demethylation level in the rat cortex and hippocampus to assess the loss of methylation. As expected, the demethylated PP2Ac levels were increased significantly in the 4-week and 16-week Mn-exposed group compared with the control groups (Fig. 5e-h). However, methionine intervention significantly reduced demethylated PP2Ac expression levels, which almost reached the levels in control rats (Fig. 5e-h). The total tau and PP2Ac levels in each group showed no significant difference. These data suggested that additional methionine intervention could avoid tau hyperphosphorylation by avoiding excessive loss of PP2Ac methylation in the rat models of subacute and chronic Mn exposure.

\section{Methionine Prevents LCMT-1 and PME-1 Expression Imbalance by Maintaining Hippocampal SAM Levels in Mn-Exposed Rats}

To confirm that methionine prevents Mn-induced PP2A inactivation by affecting these upstream regulators in vivo, we measured the levels of LCMT-1 and PME1 in the cortex and hippocampus of the rats. We found that LCMT-1 expression was significantly reduced in the Mn-exposed group compared with the control group, except in the 4-week cortex, which showed only a downward trend without statistical significance. In contrast, the PME-1 level in Mn-exposed rats was increased significantly. The increase or decrease in these two enzymes was more significant as the $\mathrm{Mn}$ exposure time increased (Fig. 6a-d). However, the methionine intervention group showed higher LCMT-1 and lower PME-1 levels than normal rats, similar to the Mnexposed group (Fig. 6a-d). We further measured the SAM and SAH levels in the hippocampus of rats. Mn injection resulted in time-dependent decreases in local SAM levels and the SAM/SAH ratio. The intervention of methionine during Mn exposure effectively improved the levels of SAM in the hippocampus of rats (Fig. $6 \mathrm{e}, \mathrm{f})$. These data suggested that extra methionine could avoid Mn-induced abnormal PP2Ac demethylation by upregulating LCMT-1 expression and downregulating PME-1 expression, and these alterations may be related to maintaining normal SAM levels in the hippocampal area in the Mn-exposed rat model.

\section{Preventing PP2Ac Methylation Loss Via ABL127 Mitigates the Toxic Effects of Mn on N2a Cells Even Without Exogenous Methionine}

We confirmed by in vitro and in vivo experiments that it was extremely unfavorable for nerve cells to cope with Mn toxicity without methionine supplementation, and the SAM availability in the Mn-exposed rat hippocampus was restricted. The PP2A methylation pattern is determined by the availability of methionine or SAM, which we consider to be the core molecular mechanism of Mn neurotoxicity. To show this, ABL127, a newly discovered agent that activates PP2A by competitively inhibiting PME-1 [47], was used in a neurological disease study for the first time. The N2a cells cultured without methionine were treated with $0.5 \mathrm{nM}$ ABL127 alone or in combination with $\mathrm{Mn}$ for $24 \mathrm{~h}$, and cells showed higher cell viability at this concentration (Fig. S3).

LCMT-1 expression had an upward trend after ABL127 treatment in Mn-exposed cells, and the difference was statistically significant at $1000 \mu \mathrm{M} \mathrm{Mn}$ exposure, while the expression of PME-1 did not change obviously (Fig. 7a-c). As expected, ABL127 treatment almost completely inhibited tau hyperphosphorylation by maintaining the PP2Ac hypermethylation status in Mn-exposed cells (Fig. $7 \mathrm{~d}-1$ ), increased the ratio of GSH to GSSG, and reduced intracellular ROS levels, especially in cells exposed to high concentrations of Mn (Fig. 8a-c). Most importantly, the addition of ABL127 partially alleviated cell viability damage and completely prevented apoptosis in Mn-exposed cells (Fig. $8 \mathrm{~d}-\mathrm{g}$ ). These data highlight the great potential 
a

Cortex

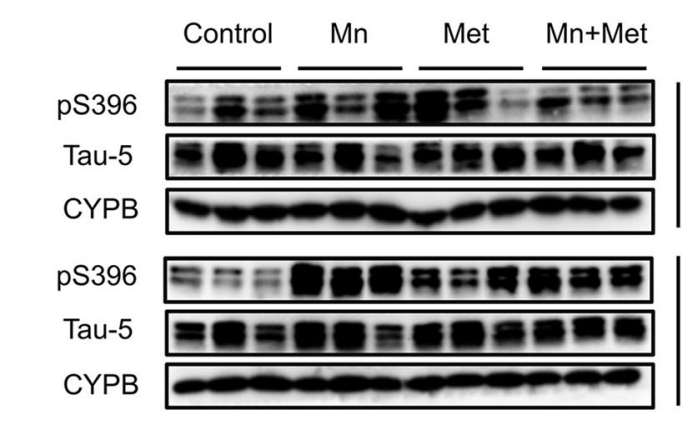

C

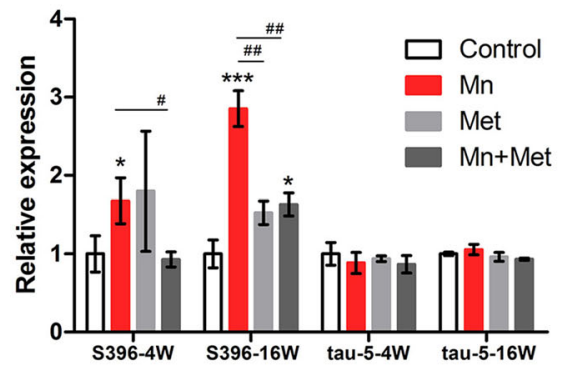

$\mathbf{e}$

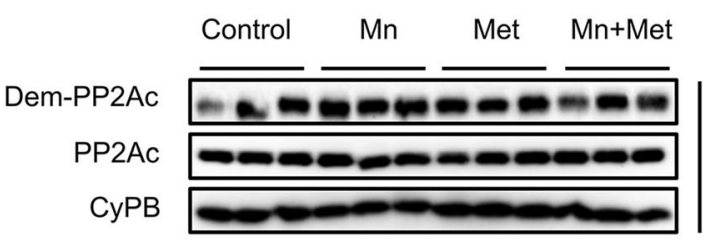

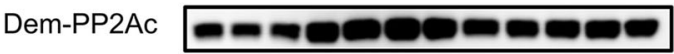

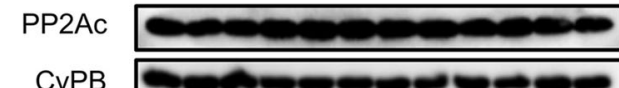

g

Cortex

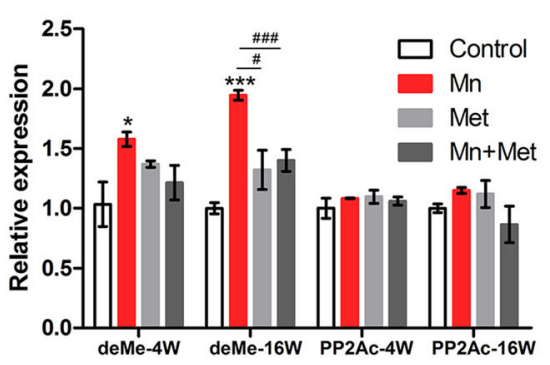

Fig. 5 Methionine treatment prevented tau hyperphosphorylation levels at a specific site by reducing PP2Ac methylation loss in the brain of Mnexposed rats. Cortical and hippocampal protein levels of total tau and ptau at Ser396 in 4-week and 16-week experiments were measured by WB (a-d). Hippocampal and cortical protein levels of total PP2Ac and demethylated PP2Ac in 4-week and 16-week experiments were measured by WB $(\mathbf{e}-\mathbf{h})$. In (c), for S396, 4 weeks: $F(3,8)=1.102, p=$ $0.403,16$ weeks: $F(3,8)=19.352, p=0.001$; for tau-5, 4 weeks: $F(3$, $8)=0.278, p=0.840,16$ weeks: $F(3,8)=1.385, p=0.316$. In (d), for S396, 4 weeks: $F(3,8)=3.918, p=0.047,16$ weeks: $F(3,8)=20.852$, b

Hippocampus

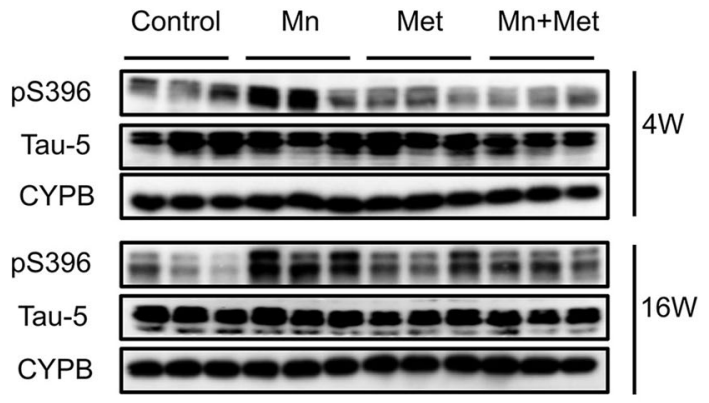

d

Hippocampus

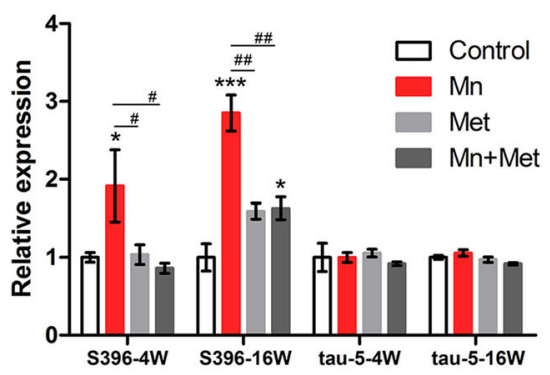

f

Hippocampus

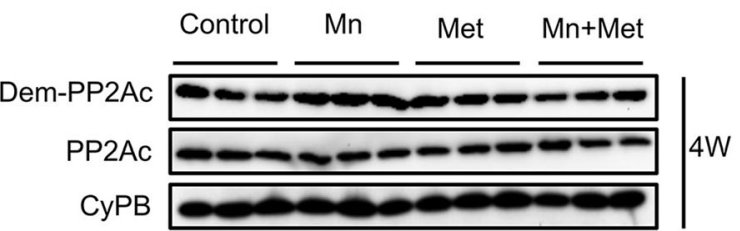

$\begin{aligned} & \text { Dem-PP2Ac } 16 \mathrm{~W} \\ & \text { PP2Ac } \\ & \text { CyPB }\end{aligned}$

h

Hippocampus

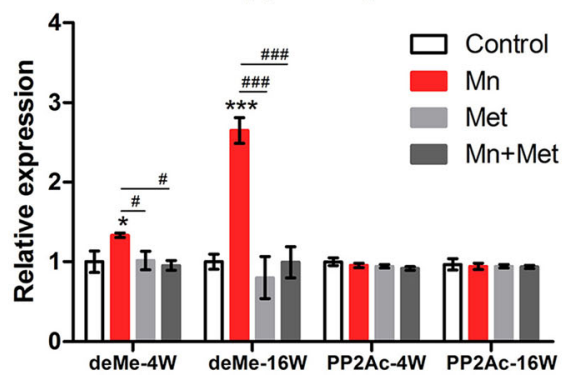

$p<0.001$; for tau-5, 4 weeks: $F(3,8)=0.320, p=0.811,16$ weeks: $F(3$, $8)=3.489, p=0.07$. In (g), for dem-PP2Ac, 4 weeks: $F(3,8)=3.598, p=$ 0.066, 16 weeks: $F(3,8)=15.630, p=0.001$; for total PP2Ac, 4 weeks: $F(3,8)=0.645, p=0.608,16$ weeks: $F(3,8)=1.762, p=0.232$. In (h), for dem-PP2Ac, 4 weeks: $F(3,8)=3.319, p=0.078,16$ weeks: $F(3,8)=$ 21.151, $p<0.001$; for total PP2Ac, 4 weeks: $F(3,8)=1.260, p=0.351$, 16 weeks: $F(3,8)=0.105, p=0.955$. One-way ANOVA, all data are expressed as means $\pm \mathrm{SD}, n=3 . * p<0.05$ and $* * * p<0.005$ versus the control group; ${ }^{\#} p<0.05,{ }^{\# \#} p<0.01$, and ${ }^{\# \#} p<0.005$ versus Mn group 
a

\section{Cortex}
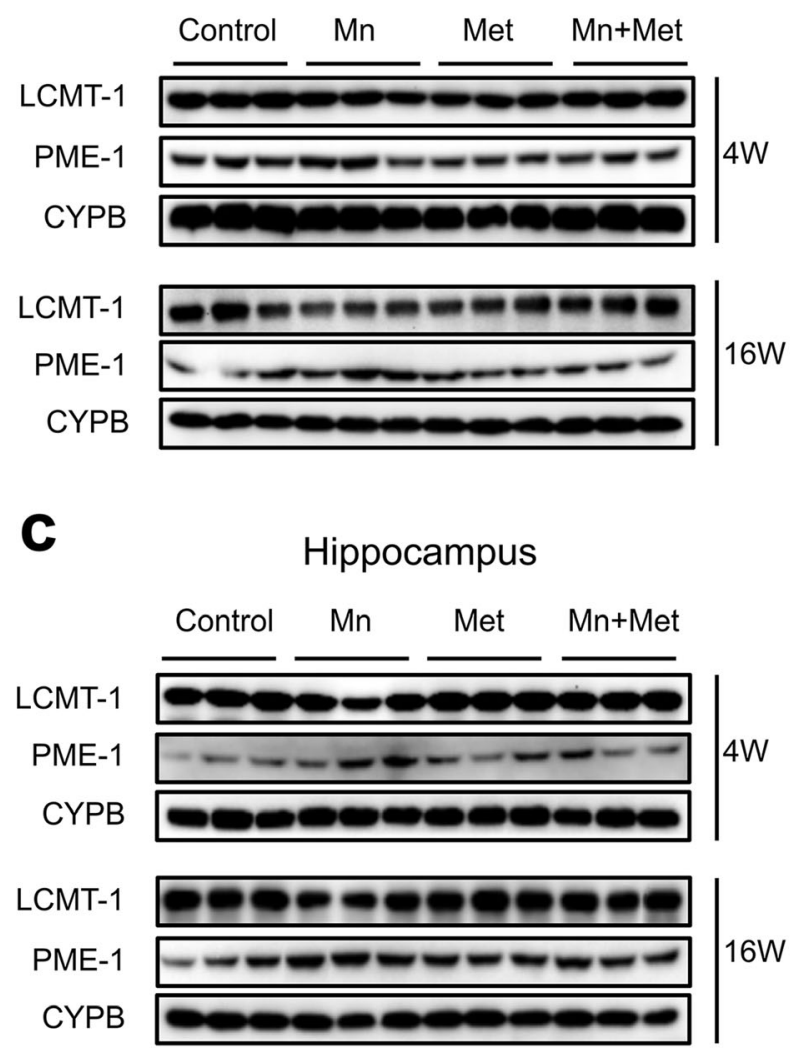

e

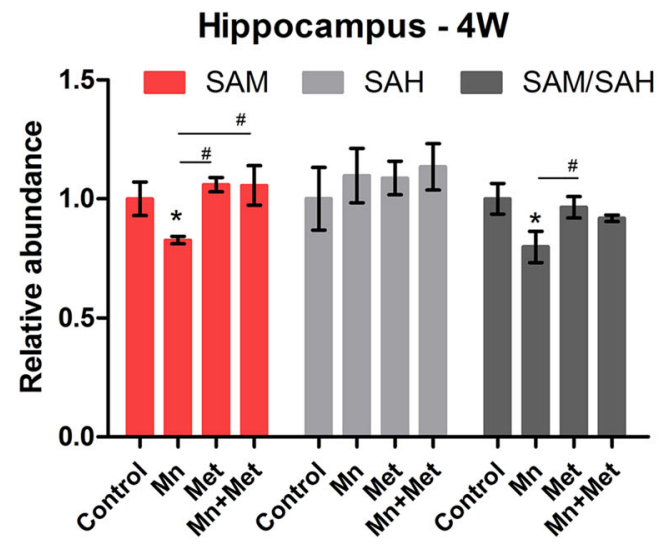

Fig. 6 Methionine regulated the expression balance between LCMT-1 and PME-1 via maintaining SAM levels in the hippocampus in Mnexposed rats. Cortical and hippocampal protein levels of LCMT-1 and PME-1 in 4-week and 16-week experiments were measured by WB (ad). Methionine treatment prevented $\mathrm{Mn}$-induced reduction in hippocampal SAM levels and the ratio of SAM to SAH (e, f). In (b), for LCMT-1, 4 weeks: $F(3,8)=1.337, p=0.329,16$ weeks: $F(3,8)=$ 3.031, $p=0.093$; for PME-1, 4 weeks: $F(3,8)=3.386, p=0.075$, 16 weeks: $F(3,8)=8.121, p=0.008$. In $(\mathbf{d})$, for LCMT-1, 4 weeks:

of PP2Ac methylation as a therapeutic target for Mn poisoning and provide theoretical support for ABL127 in the b

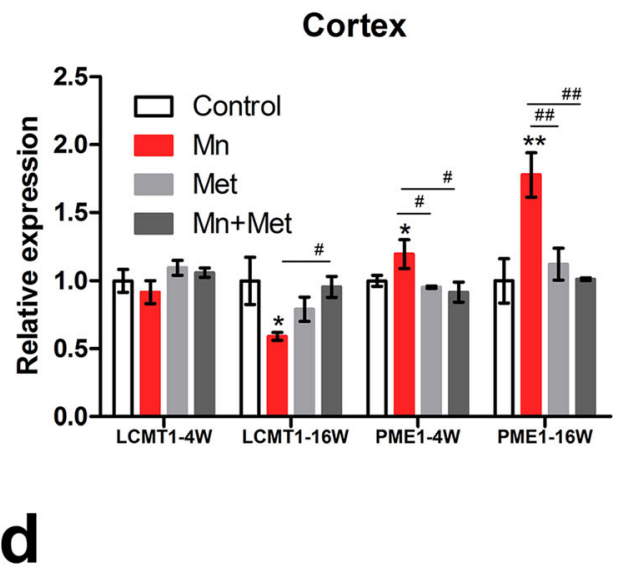

\section{Hippocampus}

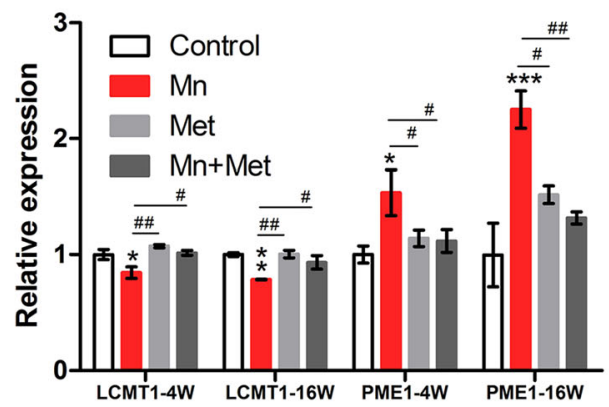

f

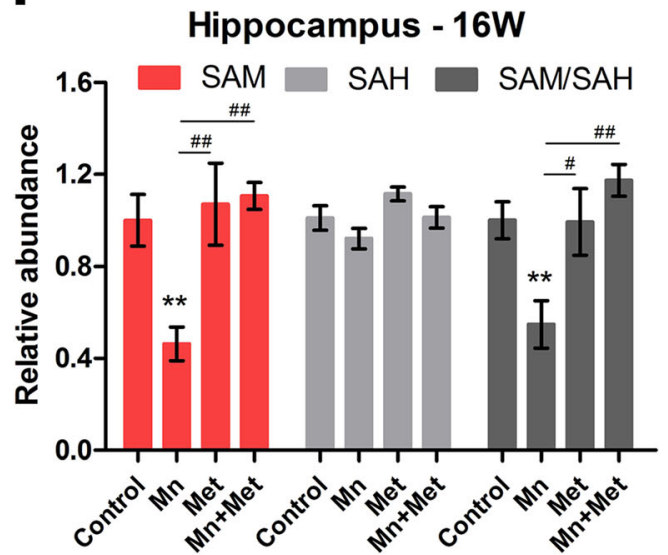

$F(3,8)=7.843, p=0.009,16$ weeks: $F(3,8)=8.847, p=0.006$; for PME-1, 4 weeks: $F(3,8)=3.648, p=0.064,16$ weeks: $F(3,8)=$ $10.268, p=0.004$. In (e), for SAM, $F(3,8)=2.097, p=0.179$; for SAH, $F(3,8)=0.286, p=0.835$; for SAM $/ \mathrm{SAH}, F(3,8)=2.890, p=0.102$. In (f), for SAM, $F(3,8)=6.792, p=0.014$; for SAH, $F(3,8)=1.490, p=$ 0.289 ; for SAM/SAH, $F(3,8)=7.386, p=0.011$. One-way ANOVA, all data are expressed as means $\pm \mathrm{SD}, n=3 . * p<0.05, * * p<0.01$, and ${ }^{* * *} p<0.005$ versus the control group; ${ }^{\#} p<0.05$ and ${ }^{\#} p<0.01$ versus Mn group

clinical treatment of neurotoxic mechanism-related diseases such as AD. 


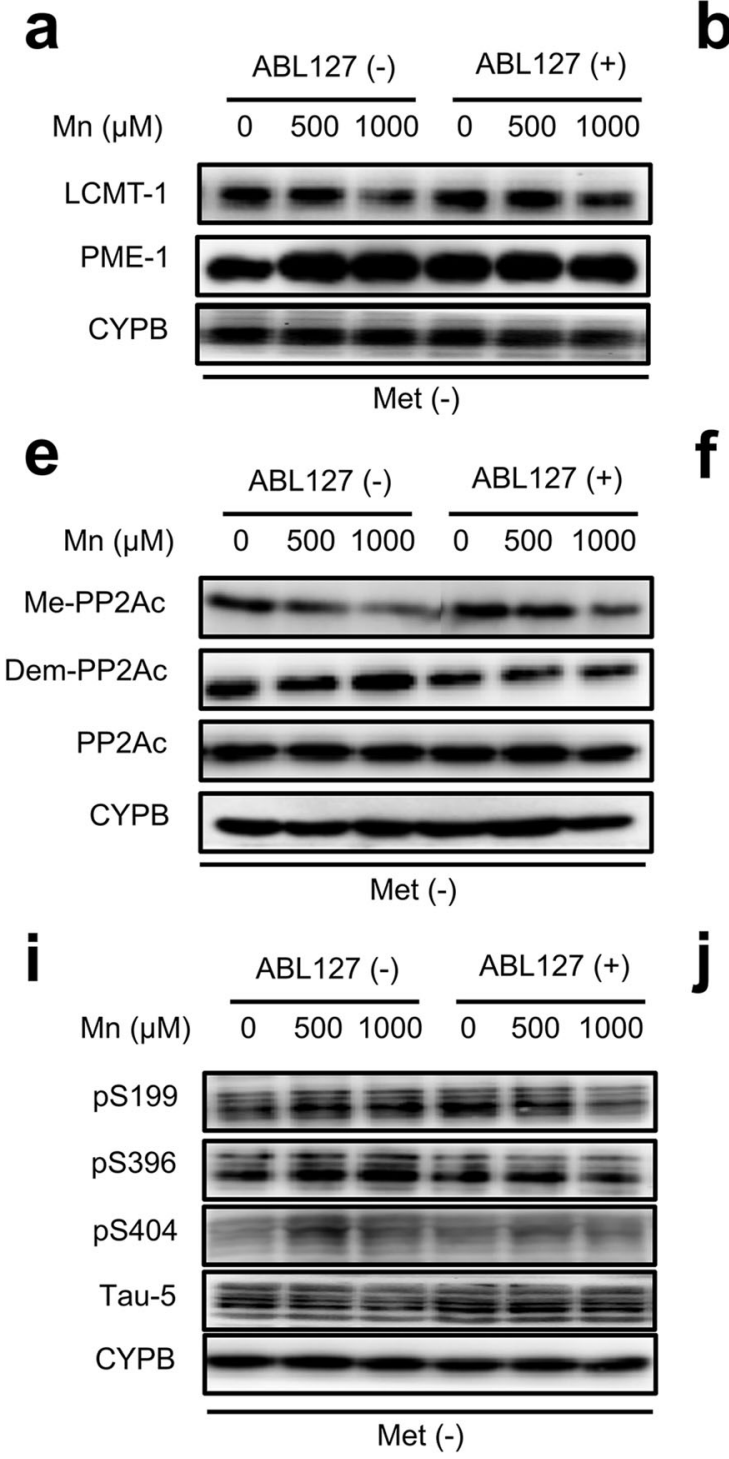

Fig. 7 ABL127 prevented hyperphosphorylation of tau by inhibiting loss of PP2Ac methylation in Mn-exposed N2a cells. Cellular protein levels of LCMT-1 and PME-1 were measured by WB $(\mathbf{a}-\mathbf{c})$. Cellular protein levels of total PP2Ac, methylated PP2Ac, and demethylated PP2Ac were measured by WB (d-g). Cellular protein levels of total tau and p-tau at Ser199, Ser396, and Ser404 were measured by WB $(\mathbf{h}-\mathbf{l})$. All data are presented as mean $\pm \mathrm{SD}, n=3$. Student's $t$ test, $* * p<0.01$ and

\section{Discussion}

The role of heavy metals in the pathogenesis of neurodegenerative diseases has been followed with growing interest. Overexposure to $\mathrm{Mn}$ is considered a risk factor for neurodegenerative diseases, characterized by a progressive motor functional neurological disorder analogous to PD [48]. However, accumulating evidence has also hinted at an association between $\mathrm{Mn}$ exposure and AD-like neuropathological changes and cognitive impairment [49]. Mn has been shown to accumulate mostly in the subcortical structures of the basal ganglia, especially the globus pallidus, substantia nigra, and
C

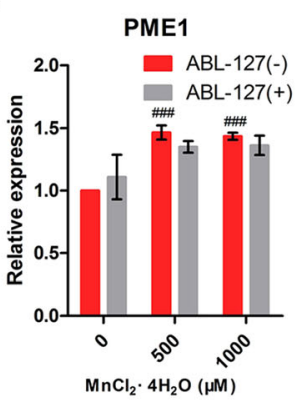

g

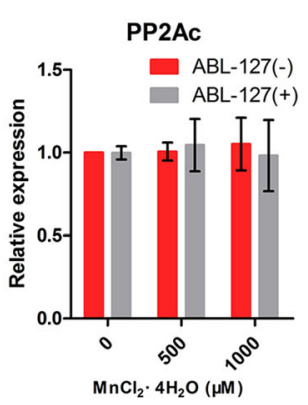

h
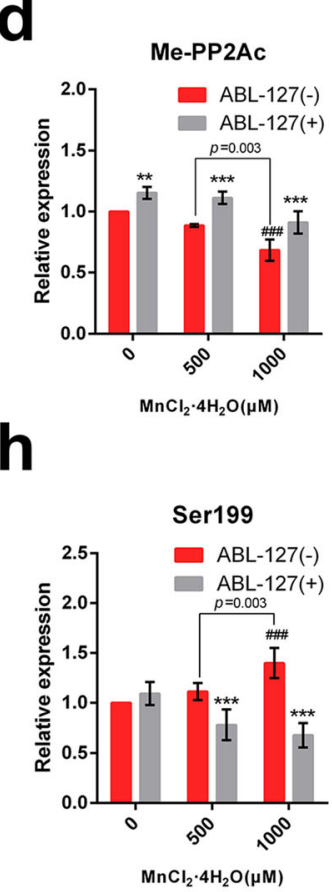

k

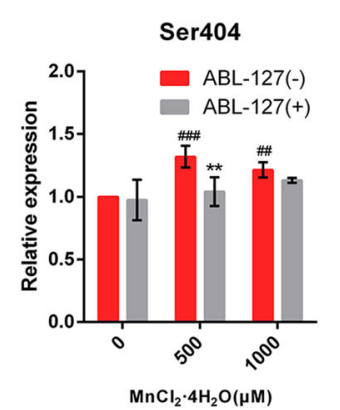

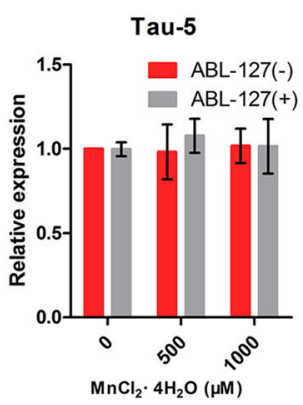

*** $p<0.005$ versus the ABL127 (-) group at the equal Mn treatment concentration. One-way ANOVA, in (b), $F(2,6)=8.853, p=0.016$; in (c), $F(2,6)=50.238, p<0.001$; in (d), $F(2,6)=29.463, p=0.001$; in (f), $F(2,6)=58.551, p<0.001$; in $(\mathbf{h}), F(2,6)=14.969, p=0.005$; in $(\mathbf{j}), F(2$, $6)=3.194, p=0.114$; in $(\mathbf{k}), F(2,6)=22.312, p=0.002 ;{ }^{\#} p<0.05$, ${ }^{\# \#} p<0.01$, and ${ }^{\# \# \#} p<0.005$ versus ABL127 (-) control group

striatum $[50,51]$. Herein, we demonstrate that Mn could also accumulate in the hippocampus of rats upon subacute (4week) and chronic (16-week) exposure and affect spatial learning and memory in a time-dependent fashion.

Alterations in the status of $\mathrm{Mn}$ are related to altered neurophysiology and cognition in humans, and either overexposure or insufficiency can lead to neurological disorders [52]. Abnormal PP2Ac demethylation is a biological process in the brains of $\mathrm{AD}$ patients [41, 53], and we found here that excessive Mn-induced neurotoxicity in vivo and in vitro was also characterized by increased PP2Ac demethylation levels. Considerable evidence suggests that the demethylation of the 
a

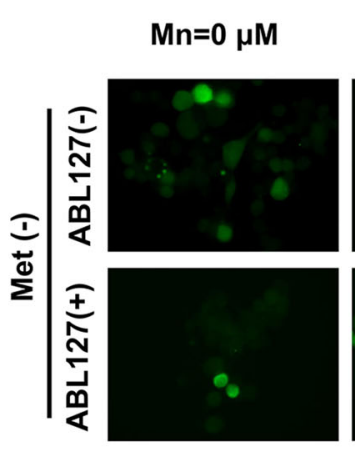

d

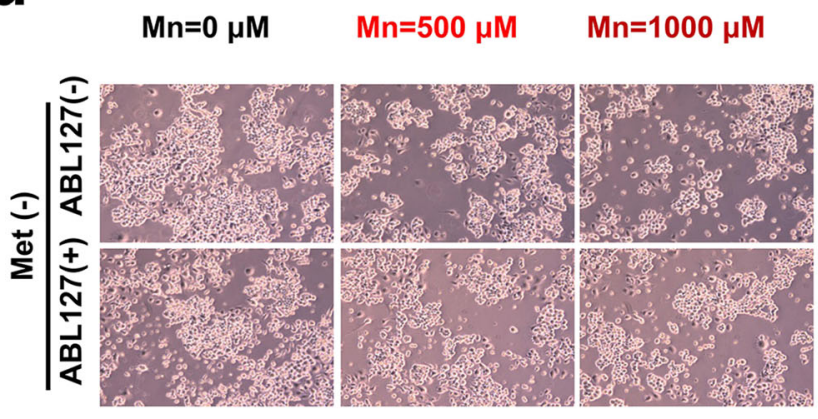

f
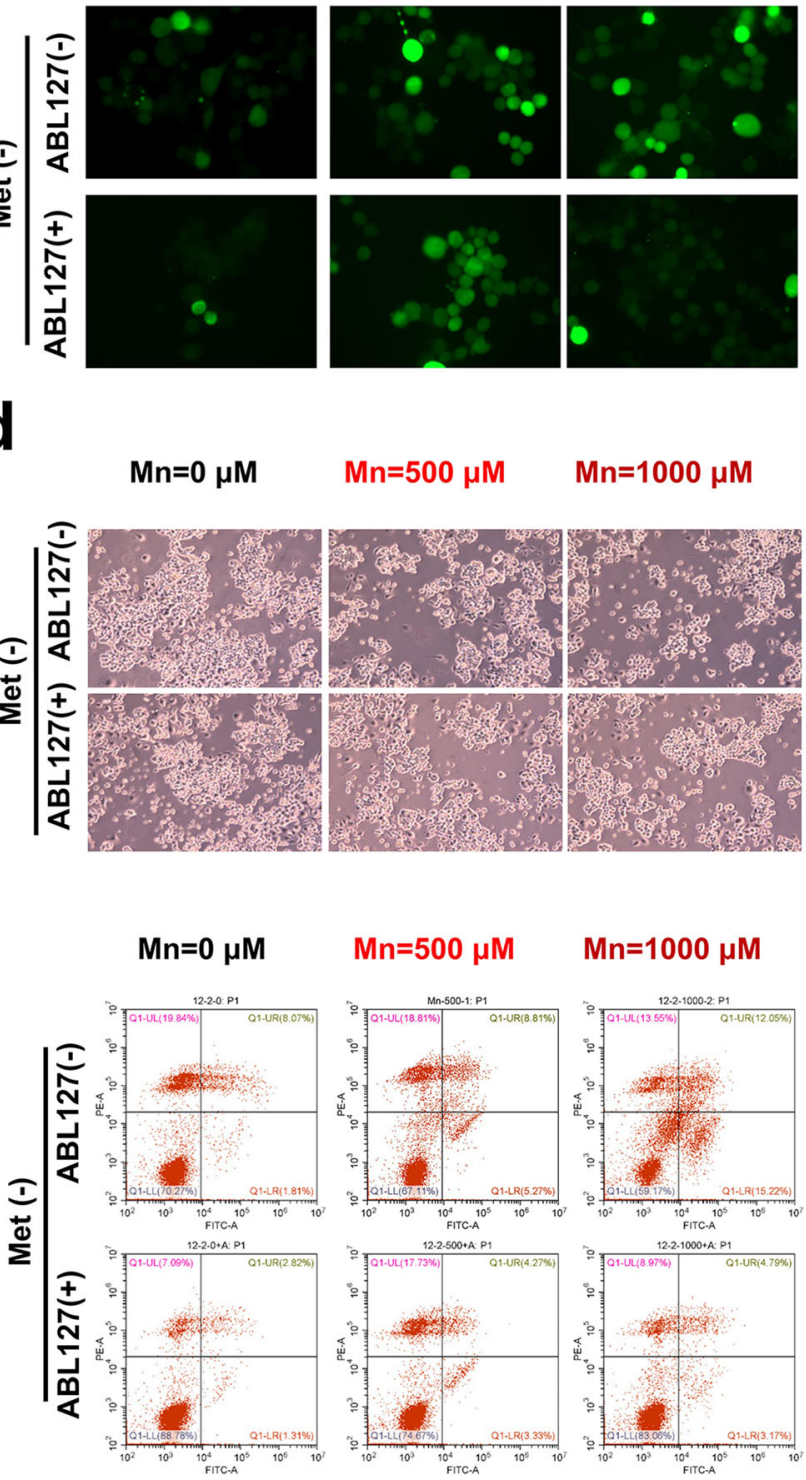

\section{e}

Fig. 8 ABL127 attenuated Mn-induced oxidative stress and partially reduced apoptosis and cell viability damage in the absence of exogenous methionine. Fluorescence image of ROS in N2a cells after Mn exposure and ABL127 treatment (a). ABL127 treatment reduced Mn-induced dramatic increase of intracellular ROS (b). ABL127 treatment increased the ratio of GSH to GSSG in Mn-induced N2a cells (c). Morphology images of N2a cells after Mn exposure and ABL127 treatment (d). ABL127 treatment attenuated Mn-induced impaired cell

carboxyl group in PP2A AC dimers can hinder the association of $\mathrm{B} 55 \alpha$ subunits to form the PP2A holoenzyme and even reduce B55 $\alpha$ levels, leading to lower PP2A activity and abnormal tau phosphorylation $[54,55]$. This suggests that the promotion of PP2Ac methylation could prevent excessive accumulation of phosphorylated tau. However, other investigations showed that lower levels of Mn can activate PP2A [29, 56], which also suggests an inverted U-shaped connection

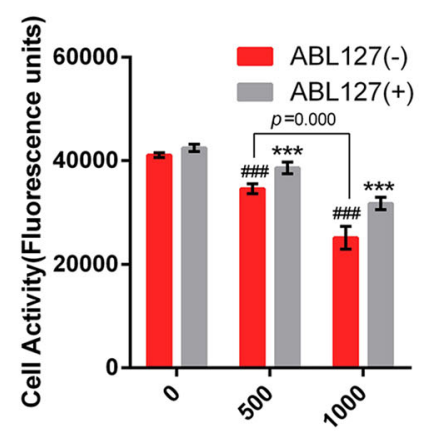

$\mathrm{MnCl}_{2} \cdot 4 \mathrm{H}_{2} \mathrm{O}(\mu \mathrm{M})$

g

b

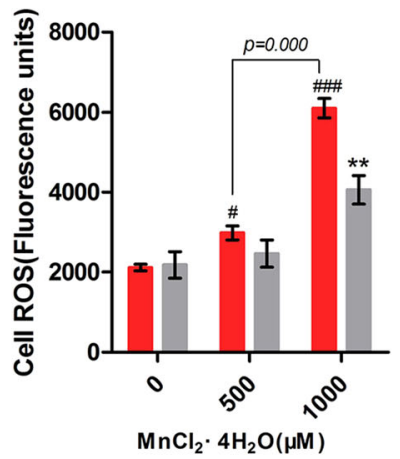

C
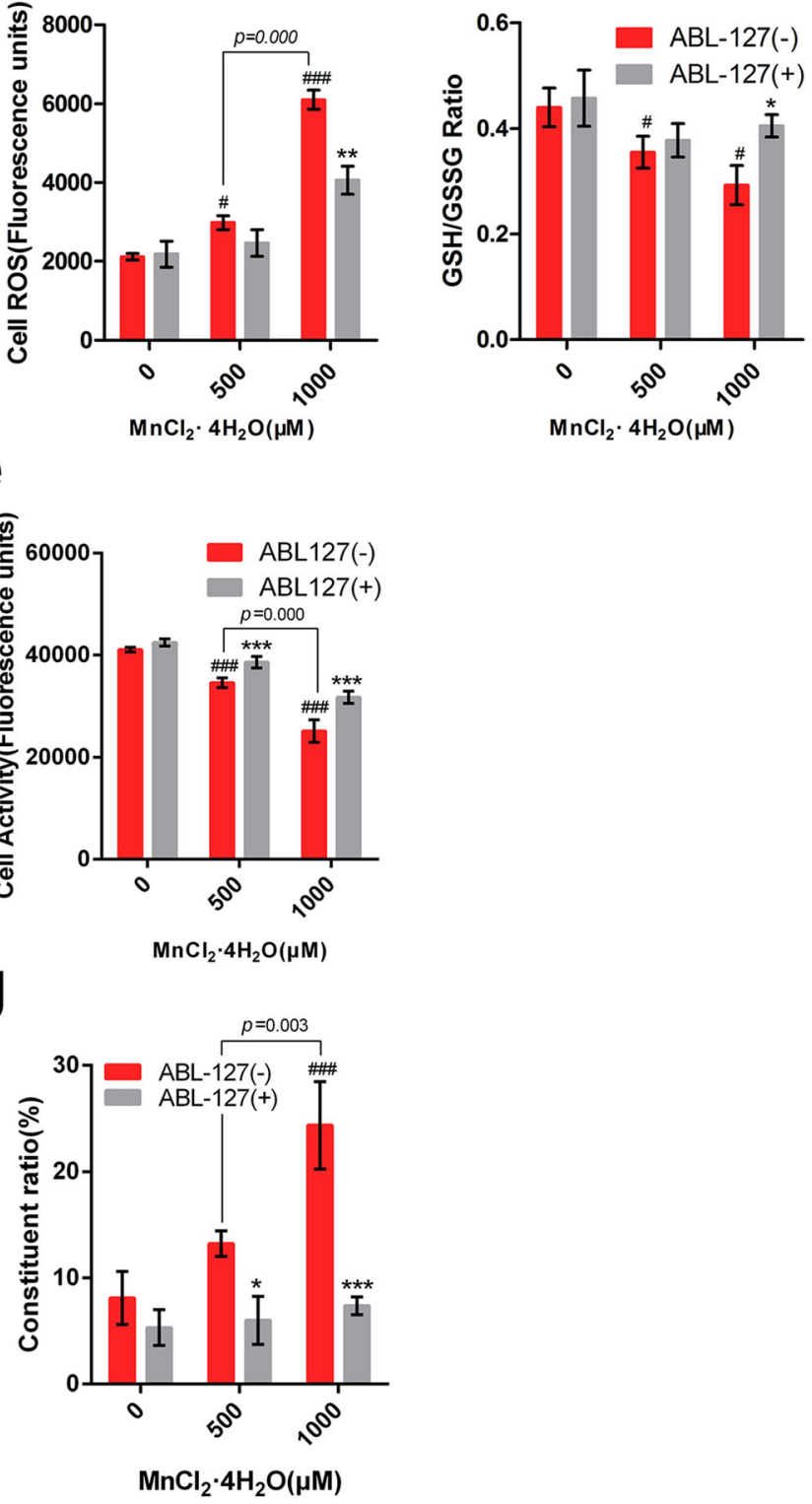

viability (e). ABL127 treatment attenuated Mn-induced apoptosis (f, g). The data shown represent the mean $\pm \mathrm{SD}$. Student's $t$ test, $* p<0.05$ and $* * p<0.01$ versus the ABL127 (-) group at the equal Mn treatment concentration. One-way ANOVA, in $(\mathbf{b})$, for $\operatorname{ROS}(n=4), F(2,9)=$ 134.762, $p<0.001$; in $(\mathbf{c})$, for GSH/GSSG $(n=4), F(2,9)=4.528$, $p=0.044$; in (e), for cell viability $(n=3), F(2,6)=59.362, p<0.001$; in $(\mathrm{g})$, for apoptosis $(n=3), F(2,6)=65.396, p<0.001$; ${ }^{\#} p<0.05$, ${ }^{\# \#} p<0.01$, and ${ }^{\# \# \#} p<0.005$ versus ABL127 (-) control group

between manganese exposure and neurological dysfunction. As a hallmark of tau pathology, almost 20 threonine and serine residues have been found to be associated with normal and physiologically important phosphorylation processes $[57,58]$. PP2A has previously been shown to dephosphorylate p-tau in vitro at Ser202, Ser262, and Ser356 sites that are hyperphosphorylated in AD [59]. We found that dephosphorylation at Ser199, Ser396, and Ser404 may also be regulated 
by PP2A in our current Mn exposure model. The phosphorylation of these three tau sites was considered to be a sign that can predict the transition of mild cognitive dysfunction to $\mathrm{AD}$ $[60,61]$, suggesting that the recovery of PP2Ac methylation may be the key to the early intervention of cognitive impairment in Mn-induced tauopathy. However, this study did not reveal the activity of the PP2A holoenzyme or the level of the $\mathrm{B}$ subunit, although an obvious association between the abnormal methylation status of PP $2 A c$ and the hyperphosphorylation of tau was observed in Mn exposure. Notably, a previous study showed that methylation significantly increased the phosphatase activity of AC dimers on neurodegenerative-related substrates in the absence of B $55 \alpha$ [62].

In this study, methionine promoted SAM-dependent PP2Ac methylation and played a rescue role in Mn neurotoxicity. Emerging studies have linked SAM metabolic disorders to diseases of the nervous system [63-65], and decreased levels of SAM were recently detected in the cerebrospinal fluid of AD patients [66]. We provided evidence that local SAM availability could be the most critical determinant in Mn-induced PP2Ac methylation pattern alteration. Our study provides comprehensive data on PP2Ac methylation changes induced by alterations in SAM levels in a Mn-poisoning neuropathic process. We found that in the rat cortex and hippocampus, PP2Ac demethylation during Mn exposure correlated with a decrease in SAM concentration, and importantly, the elevation of local SAM levels through the injection of methionine in vivo ensured normal PP2Ac methylation status and significantly improved tau-related cognitive impairment in rats. In addition, a marked reduction in LCMT-1 and an increase in PME-1 were observed in our Mn-exposed models in vitro and in vivo. The simultaneous abnormal expression of these two key enzymes regulating PP2Ac methylation has also been confirmed to occur in multiple pathological processes, including $\mathrm{AD}[28,67,68]$. Past studies have confirmed that long-term SAM deficiency or elevated SAH will inhibit not only the activity but also the expression of LCMT-1 in neuronal cells, and our data emphasize the link between LCMT-1 expression and the SAM/SAH ratio in Mn neurotoxicity [69]. There are also emerging studies showing that the expression of LCMT-1 and PME-1 may be regulated by posttranscriptional regulation of miRNA-195 [70]. Future work should focus on verifying the mechanism underlying the decreased LCMT-1 and increased PME-1 regulated by Mn, especially the effects of SAM.

Moreover, aza- $\beta$-lactams (ABLs) are a class of recently screened inhibitors that can selectively inhibit PME-1, which inhibits PP2Ac demethylation and thereby increases PP2A activity [47]. Our in vitro results showed that ABL127 prevents Mn-induced excessive demethylation of PP2Ac to avoid the hyperphosphorylation of tau, which largely relieved the ROS surge and completely inhibited cell apoptosis. In short, our study highlights the critical roles of methionine/SAM and PP2Ac methylation patterns in resistance to Mn neurotoxicity and the potential value of ABL application in tau-related neurodegenerative lesions.

Previous studies have shown that the accumulation of $\mathrm{Mn}$ in the basal ganglia area can cause ROS bursts as well as increased GSH consumption and thiol depletion [71]. However, the exact pathway leading to the generation of ROS by Mn is still unknown. The NFTs formed by hyperphosphorylated tau can inhibit mitochondrial transport, induce the production of cytotoxic ROS, and cause neuronal apoptosis [22], and a study recently indicated that the accumulation of tau aggregates on the membrane may induce a calcium influx that triggers ROS production in NADPH oxidase, leading to neuronal cell death [72]. We found here that PP2Ac methylation continuously activated by exogenous methionine or ABL127 significantly inhibited ROS levels, increased the GSH/GSSG ratio, and prevented the loss of cell viability and apoptosis in Mn-exposed N2a cells, and this series of changes in oxidative damage indicators may be partly attributed to the recovery of the direct control of phosphorylated tau by PP2A. These results are consistent with our previous report that the promotion of the methylation of PP2Ac by the overexpression of LCMT-1 reduced oxidative stress and protected cell viability [73].

The synthesis of SAM is catalyzed by methionine adenosyltransferase (MAT) using L-methionine and adenosine triphosphate (ATP) [74]. Here, we found that excess Mn caused low SAM levels in N2a cells and the rat hippocampus. The cysteine residue of MAT can be covalently modified under oxidant stress, leading to MAT inactivation which is also related to a decrease in the ratio of GSH to GSSG [75, 76]. Recently, it was shown that excess ROS caused a large amount of homocysteine to flow into the transsulfuration pathway to generate $\mathrm{GSH}$, thereby reducing the production of endogenous methionine [77]. In addition, Mn-induced oxidative stress can damage mitochondrial ATP synthesis [71]. This evidence indicates that ROS may be a mediator of the Mninduced decline in SAM availability (Fig. 9).

Multiple studies have concluded that oxidative stress and mitochondrial dysfunction may be early events in tauopathies [39]. Excess ROS may promote tau phosphorylation through the inactivation of PP2A or the activation of tau kinase and accelerate the accumulation of $\mathrm{p}$-tau due to lipid peroxidation of the cell membranes [40]. Our results showed that the complete inhibition of Mn-induced tau phosphorylation by maintaining PP2Ac methylation partially reduced intracellular ROS levels, suggesting that Mn may promote the generation of ROS in other ways. For example, a recent study showed that $\mathrm{Mn}$ can cause the accumulation of toxic $\mathrm{Fe}^{2+}$ through the translational repression of amyloid precursor protein (APP) and $\mathrm{H}$-ferritin, thereby generating ROS [78]. If so, based on previous findings, oxidative stress 


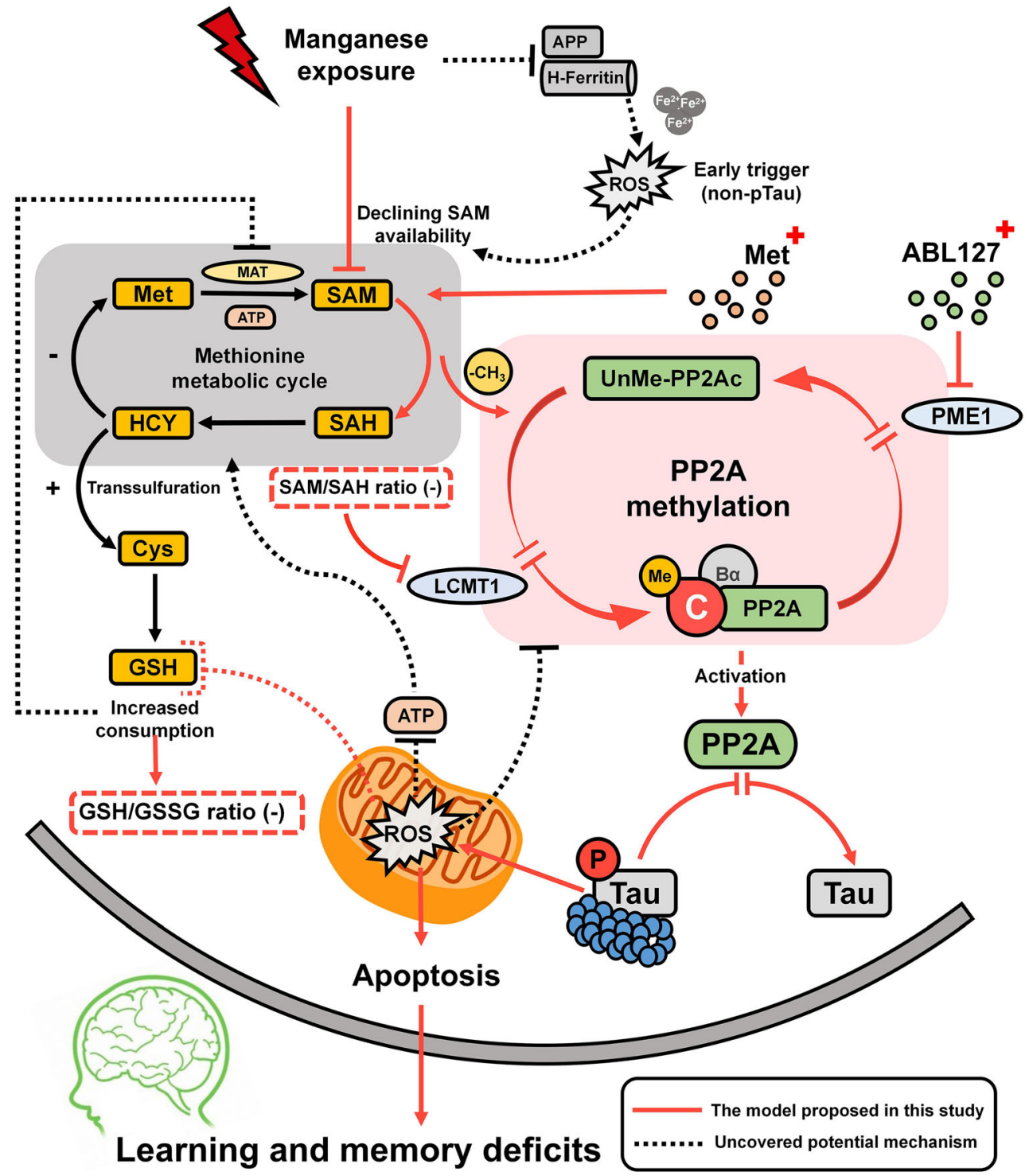

Fig. 9 Proposed potential model of Mn-induced tauopathy and treatment targets. Based on previous studies and this work, the hypothesis of a vicious circle between oxidative stress and tau phosphorylation in manganese $(\mathrm{Mn})$ poisoning is proposed. Mn exposure leads to a surge in methionine requirements and impaired SAM availability. The restricted SAM/SAH ratio limits the methylation of PP2Ac via unbalanced expression of LCMT-1 and PME-1. The resulting inactivated PP2A blocks the dephosphorylation of the substrate p-tau, leading to abnormal $\mathrm{p}$-tau accumulation and increased ROS production. The p-tau-induced excessive ROS further decreases SAM levels by inhibiting MAT activity and ATP synthesis as well as the resynthesis of

may be an early trigger for the decline in local SAM availability in Mn neurotoxicity (Fig. 9). The resulting hypomethylation of PP2Ac and tau phosphorylation then continue to stimulate the surge in ROS. Moreover, a previous study indicated that oxides may bind to the thiol pair of PP2Ac, prompting PP2A inactivation [79]. Another report showed that oxidative stress could regulate PP2Ac demethylation by affecting the distribution of PME-1 in cells without changing methionine and then continues to disrupt the methionine cycle and PP2A methylation. These factors aggravate the phosphorylation of tau and subsequently promote the explosive production of ROS. Continuous oxidative stress leads to nerve cell apoptosis, which ultimately causes AD-like learning and memory impairment. The ROS caused by non-ptau factors, such as the accumulation of $\mathrm{Fe}^{2+}$ caused by inhibiting the expression of APP and $\mathrm{H}$-ferritin in the early stage of Mn exposure, may first trigger the decline in SAM availability. Exogenous methionine or ABL127 supplementation activates PP2Ac methylation by increasing SAM levels or inhibiting PME-1, thereby rescuing Mn neurotoxicity

protein expression [80]. Therefore, a complicated pathological positive feedback loop may be formed between ROS and p-tau, eventually causing irreversible neuronal damage (Fig. 9). Antioxidants have been reported to reduce Mn-induced DNA damage in SH-SY5Y cells [81], but we have not yet applied antioxidants to the Mn exposure model in this study, which may help verify the leading role of ROS in Mn neurotoxicity. 
For the prevention and treatment of Mn poisoning and other degenerative disorders with similar mechanisms, we demonstrated that exogenous methionine supplementation could effectively prevent Mn-induced tau hyperphosphorylation in cell and animal models by increasing the levels of PP2Ac methylation, thereby reducing oxidative stress and cell damage as well as improving learning and memory. Furthermore, an unanticipated finding is that Mn could selectively reduce the availability of SAM in the hippocampus and increase the demand for methionine/SAM, which suggests that nutrients such as vitamin $B_{12}$, folic acid, and choline, which can increase the effectiveness of methyl use, may also help protect the brain from Mn neurotoxicity [82]. SAM levels are influenced by a variety of environmental factors including diet and diabetes [83]. SAM deficiency caused by these adverse conditions may increase the body's sensitivity to Mn neurotoxicity. In addition, ABL127 selectively interferes with PME-1 to promote PP2Ac hypermethylation and attenuates Mn-induced tau-related neurotoxic injury in vitro, playing a protective role. However, based on the few studies currently evaluating the in vivo effect of ABL127, the next stage needs to focus on investigating the administration method and the effect of ABL127 in the in vivo model. In short, SAM and PP2Ac methylation may be potential effective targets for the treatment of Mn-induced tauopathy, and most importantly, these features suggest that efforts to improve PP2A activity may inhibit the hyperphosphorylation of tau and alleviate related pathologies.

\section{Conclusion}

In conclusion, we show that Mn-induced neurotoxicity is characterized by PP2Ac demethylation accompanied by abnormally decreased LCMT-1 and increased PME-1, which are associated with tau hyperphosphorylation, cell oxidative damage, and spatial learning and memory deficits, and that the poor availability of SAM in specific brain regions that control memory is likely to play a critical role in the loss of methylation during this process. Importantly, the maintenance of local SAM levels through continuous supplementation with exogenous methionine can effectively prevent the tau-related neuropathological changes and cognitive impairment caused by PP2Ac demethylation in Mn neurotoxicity. In addition, our results also suggest the possibility of specific PP2Ac methylation activators as potential therapeutic drugs in the future. This study reveals the important roles of SAM and PP2Ac methylation as potential therapeutic targets for Mn poisoning and the connection between $\mathrm{Mn}$ and $\mathrm{AD}$ neuropathology.

Acknowledgments This work was supported by the National Natural Science Foundation of China (NSFC) grants 21567006 and 81760576 to Cailing Lu, NSFC grant 81860585 to Xiyi Li, and NSFC grant 81460506 to Shen Tang, and Guangxi Natural Science Foundation grant 2019 GXNSFGA245002 to Yunfeng Zou and grant 2017GXNSFGA198003 to Xiaobo Yang.

Required Author Forms Disclosure forms provided by the authors are available with the online version of this article.

Authors' Contributions Cailing Lu and Xiyi Li conceptualized and designed the study. Bin $\mathrm{Wu}$, Haiqing Cai, and Shen Tang performed the experiment, drafted the initial manuscript, and approved the final manuscript as submitted. Deqiang Xiao processed and analyzed data. Xinhang Wang, Yilu Xu, Qianqian Shi, Ling Meng, Ning Zhang, and Lancheng Wei coordinated the project. Yunfeng Zou and Xiaobo Yang supervised experiments and revised manuscript.

\section{Compliance with Ethical Standards}

Disclosures The authors declare that they have no competing interests.

Ethics Approval and Consent to Participate All animal studies were approved by the ethical committee for animal experiment of the Guangxi Medical University (GXMU-2014-036) and in accordance with the international standards of guidelines for the Care and Use of Laboratory Animal in animal experiments. This study strived to minimize the number and the suffering of experimental animals.

Abbreviations Met, methionine; p-tau, phosphorylated tau protein; wk, week; ROS, reactive oxygen species; GSH, glutathione; MWM, Morris water maze; AD, Alzheimer's disease; PD, Parkinson's disease; i.p., intraperitoneal; WB, Western blotting

\section{References}

1. Chen P, Chakraborty S, Mukhopadhyay S, et al. Manganese homeostasis in the nervous system. J Neurochem 2015;134(4):601610.

2. Horning KJ, Caito SW, Tipps KG, Bowman AB, Aschner M. Manganese Is Essential for Neuronal Health. Annu Rev Nutr 2015;35:71-108.

3. Hudnell HK. Effects from environmental Mn exposures: a review of the evidence from non-occupational exposure studies. Neurotoxicology. 1999;20:379-397.

4. Iregren A. Manganese neurotoxicity in industrial exposures: proof of effects, critical exposure level, and sensitive tests. Neurotoxicology. 1999;20:315-323.

5. Koretsky AP, Silva AC. Manganese-enhanced magnetic resonance imaging (MEMRI). NMR Biomed 2004;17(8):527-531.

6. Ponzoni S. Macrophages-mediated neurotoxic effects of intranigral manganese administration are attenuated by minocycline. Neurosci Lett 2012;506(1):136-140.

7. Silva AC, Bock NA. Manganese-enhanced MRI: an exceptional tool in translational neuroimaging. Schizophr Bull 2008;34(4): 595-604.

8. Racette BA, Searles Nielsen S, Criswell SR, et al. Dose-dependent progression of parkinsonism in manganese-exposed welders. Neurology. 2017;88(4):344-351.

9. Fored CM, Fryzek JP, Brandt L, et al. Parkinson's disease and other basal ganglia or movement disorders in a large nationwide cohort of Swedish welders. Occup Environ Med 2006;63(2):135-140. 
10. Bouchard MF, Sauvé S, Barbeau B, et al. Intellectual impairment in school-age children exposed to manganese from drinking water. Environ Health Perspect 2011;119(1):138-143.

11. Schneider JS, Decamp E, Koser AJ, et al. Effects of chronic manganese exposure on cognitive and motor functioning in non-human primates. Brain Res 2006;1118(1):222-231.

12. Mandelkow EM, Mandelkow E. Tau as a marker for Alzheimer's disease. Trends Biochem Sci 1993;18(12):480-483.

13. Yu Y, Run X, Liang Z, et al. Developmental regulation of tau phosphorylation, tau kinases, and tau phosphatases. J Neurochem 2009;108(6):1480-1494.

14. Iqbal K, Alonso Adel $\mathrm{C}$, Chen $\mathrm{S}$, et al. Tau pathology in Alzheimer disease and other tauopathies. Biochim Biophys Acta 2005;1739: 198-210.

15. Wang JZ, Grundke-Iqbal I, Iqbal K. Kinases and phosphatases and tau sites involved in Alzheimer neurofibrillary degeneration. Eur J Neurosci 2007;25(1):59-68.

16. Olivieri G, Brack C, Müller-Spahn F, et al. Mercury induces cell cytotoxicity and oxidative stress and increases beta-amyloid secretion and tau phosphorylation in SHSY5Y neuroblastoma cells. J Neurochem 2000;74(1):231-236.

17. Bihaqi SW, Zawia NH. Enhanced taupathy and AD-like pathology in aged primate brains decades after infantile exposure to lead $(\mathrm{Pb})$. Neurotoxicology. 2013;39:95-101.

18. Wang W, Yang Y, Ying C, et al. Inhibition of glycogen synthase kinase-3beta protects dopaminergic neurons from MPTP toxicity. Neuropharmacology. 2007;52(8):1678-1684.

19. Cai T, Che H, Yao T, et al. Manganese induces tau hyperphosphorylation through the activation of ERK MAPK pathway in PC12 cells. Toxicol Sci 2011;119(1):169-177.

20. Alonso AC, Zaidi T, Grundke-Iqbal I, Iqbal K. Role of abnormally phosphorylated tau in the breakdown of microtubules in Alzheimer disease. Proc Natl Acad Sci U S A 1994;91(12):5562-5566.

21. Alonso AC, Grundke-Iqbal I, Iqbal K. Alzheimer's disease hyperphosphorylated tau sequesters normal tau into tangles of filaments and disassembles microtubules. Nat Med 1996;2(7):783787.

22. Alavi Naini SM, Soussi-Yanicostas N. Tau Hyperphosphorylation and Oxidative Stress, a Critical Vicious Circle in Neurodegenerative Tauopathies? Oxidative Med Cell Longev 2015;2015:151979.

23. Shi Y. Serine/threonine phosphatases: mechanism through structure. Cell. 2009;139(3):468-484.

24. Janssens V, Goris J. Protein phosphatase 2A: a highly regulated family of serine/threonine phosphatases implicated in cell growth and signalling. The Biochemical journal 2001;353:417-439.

25. Virshup DM. Protein phosphatase 2A: a panoply of enzymes. Curr Opin Cell Biol 2000;12(2):180-185.

26. Tolstykh T, Lee J, Vafai S, Stock JB. Carboxyl methylation regulates phosphoprotein phosphatase $2 \mathrm{~A}$ by controlling the association of regulatory B subunits. EMBO J 2000;19(21):5682-5691.

27. Xing Y, Li Z, Chen Y, et al. Structural mechanism of demethylation and inactivation of protein phosphatase 2A. Cell. 2008;133(1):154163.

28. Park HJ, Lee KW, Oh S, et al. Protein Phosphatase 2A and Its Methylation Modulating Enzymes LCMT-1 and PME-1 Are Dysregulated in Tauopathies of Progressive Supranuclear Palsy and Alzheimer Disease. J Neuropathol Exp Neurol 2018;77(2): 139-148.

29. Gong CX, Grundke-Iqbal I, Iqbal K. Dephosphorylation of Alzheimer's disease abnormally phosphorylated tau by protein phosphatase-2A. Neuroscience. 1994;61(4):765-772.

30. Gong CX, Singh TJ, Grundke-Iqbal I, Iqbal K. Phosphoprotein phosphatase activities in Alzheimer disease brain. J Neurochem 1993;61(3):921-927.
31. Gong CX, Shaikh S, Wang JZ, et al. Phosphatase activity toward abnormally phosphorylated tau: decrease in Alzheimer disease brain. J Neurochem 1995;65(2):732-738.

32. Giulidori P, Galli-Kienle M, Catto E, Stramentinoli G. Transmethylation, transsulfuration, and aminopropylation reactions of S-adenosyl-L-methionine in vivo. J Biol Chem 1984;259(7):4205-4211.

33. Mato JM, Alvarez L, Ortiz P, Pajares MA. S-adenosylmethionine synthesis: molecular mechanisms and clinical implications. Pharmacol Ther 1997;73(3):265-280.

34. Fuso A, Seminara L, Cavallaro RA, D'Anselmi F, Scarpa S. Sadenosylmethionine/homocysteine cycle alterations modify DNA methylation status with consequent deregulation of PS1 and BACE and beta-amyloid production. Mol Cell Neurosci 2005;28(1):195-204.

35. C. Tapia-Rojas, C. B. Lindsay, C. Montecinos-Oliva, et al. Is Lmethionine a trigger factor for Alzheimer's-like neurodegeneration?: Changes in Abeta oligomers, tau phosphorylation, synaptic proteins, Wnt signaling and behavioral impairment in wild-type mice. Mol Neurodegener 2015;10:62.

36. Bonilla E, Arrieta A, Castro F, Dávila JO, Quiroz I. Manganese toxicity: free amino acids in the striatum and olfactory bulb of the mouse. Investig Clin 1994;35(4):175-181.

37. O'Connor CM, Perl A, Leonard D, Sangodkar J, Narla G. Therapeutic targeting of PP2A. Int J Biochem Cell Biol 2018;96: 182-193.

38. Anderson ME. Determination of glutathione and glutathione disulfide in biological samples. Methods Enzymol 1985;113:548-555.

39. Singh A, Kukreti R, Saso L, Kukreti S. Oxidative Stress: A Key Modulator in Neurodegenerative Diseases. Molecules (Basel, Switzerland). 2019;24(8):1583.

40. Tönnies E, Trushina E. Oxidative Stress, Synaptic Dysfunction, and Alzheimer's Disease. J Alzheimer's Disease : JAD 2017;57(4): 1105-1121.

41. Sontag JM, Sontag E. Protein phosphatase 2A dysfunction in Alzheimer's disease. Front Mol Neurosci 2014;7:16.

42. Sontag JM, Nunbhakdi-Craig V, Sontag E. Leucine carboxyl methyltransferase 1 (LCMT1)-dependent methylation regulates the association of protein phosphatase $2 \mathrm{~A}$ and Tau protein with plasma membrane microdomains in neuroblastoma cells. J Biol Chem 2013;288(38):27396-27405.

43. Kaur A, Denisova OV, Qiao X, et al. PP2A Inhibitor PME-1 Drives Kinase Inhibitor Resistance in Glioma Cells. Cancer Res 2016;76(23):7001-7011.

44. Varela-Rey M, Iruarrizaga-Lejarreta M, Lozano JJ, et al. Sadenosylmethionine levels regulate the schwann cell DNA methylome. Neuron. 2014;81(5):1024-1039.

45. Smith ZD, Meissner A. DNA methylation: roles in mammalian development. Nat Rev Genet 2013;14(3):204-220.

46. Martin L, Latypova X, Wilson CM, et al. Tau protein phosphatases in Alzheimer's disease: the leading role of PP2A. Ageing Res Rev 2013;12(1):39-49.

47. Bachovchin DA, Mohr JT, Speers AE, et al. Academic crossfertilization by public screening yields a remarkable class of protein phosphatase methylesterase-1 inhibitors. Proc Natl Acad Sci U S A 2011;108(17):6811-6816

48. Gunter TE, Gerstner B, Gunter KK, et al. Manganese transport via the transferrin mechanism. Neurotoxicology. 2013;34:118-127.

49. Calderón-Garcidueñas L, Serrano-Sierra A, Torres-Jardón R, et al. The impact of environmental metals in young urbanites' brains. Exp Toxicol Pathol : official journal of the Gesellschaft fur Toxikologische Pathologie 2013;65(5):503-511.

50. Newland MC, Ceckler TL, Kordower JH, Weiss B. Visualizing manganese in the primate basal ganglia with magnetic resonance imaging. Exp Neurol 1989;106(3):251-258. 
51. Kwakye GF, Paoliello MM, Mukhopadhyay S, Bowman AB, Aschner M. Manganese-Induced Parkinsonism and Parkinson's Disease: Shared and Distinguishable Features. Int J Environ Res Public Health 2015;12(7):7519-7540.

52. Balachandran RC, Mukhopadhyay S, McBride D, et al. Brain manganese and the balance between essential roles and neurotoxicity. J Biol Chem 2020;295(19):6312-6329.

53. Basurto-Islas G, Blanchard J, Tung YC, et al. Therapeutic benefits of a component of coffee in a rat model of Alzheimer's disease. Neurobiol Aging 2014;35(12):2701-2712.

54. Sontag E, Luangpirom A, Hladik C, et al. Altered expression levels of the protein phosphatase $2 \mathrm{~A} \mathrm{ABalphaC}$ enzyme are associated with Alzheimer disease pathology. J Neuropathol Exp Neurol 2004;63(4):287-301

55. Yu XX, Du X, Moreno CS, et al. Methylation of the protein phosphatase 2A catalytic subunit is essential for association of Balpha regulatory subunit but not SG2NA, striatin, or polyomavirus middle tumor antigen. Mol Biol Cell 2001;12(1):185-199.

56. Zhang D, Kanthasamy A, Anantharam V, Kanthasamy A. Effects of manganese on tyrosine hydroxylase $(\mathrm{TH})$ activity and $\mathrm{TH}$ phosphorylation in a dopaminergic neural cell line. Toxicol Appl Pharmacol 2011;254(2):65-71.

57. Hasegawa M, Morishima-Kawashima M, Takio K, et al. Protein sequence and mass spectrometric analyses of tau in the Alzheimer's disease brain. J Biol Chem 1992;267(24):17047-17054.

58. Morishima-Kawashima M, Hasegawa M, Takio K, et al. Prolinedirected and non-proline-directed phosphorylation of PHF-tau. J Biol Chem 1995;270(2):823-829.

59. Duka V, Lee JH, Credle J, et al. Identification of the sites of tau hyperphosphorylation and activation of tau kinases in synucleinopathies and Alzheimer's diseases. PLoS One 2013;8(9):e75025.

60. Diniz BS, Pinto Júnior JA, Forlenza OV. Do CSF total tau, phosphorylated tau, and beta-amyloid 42 help to predict progression of mild cognitive impairment to Alzheimer's disease? A systematic review and meta-analysis of the literature. World J Biol Psychiatry : the official journal of the World Federation of Societies of Biological Psychiatry 2008;9(3):172-182.

61. Ewers M, Buerger K, Teipel SJ, et al. Multicenter assessment of CSF-phosphorylated tau for the prediction of conversion of MCI. Neurology. 2007;69(24):2205-2212.

62. Lee KW, Chen W, Junn E, et al. Enhanced phosphatase activity attenuates $\alpha$-synucleinopathy in a mouse model. J Neurosci 2011;31(19):6963-6971.

63. Chen Z, Karaplis AC, Ackerman SL, et al. Mice deficient in methylenetetrahydrofolate reductase exhibit hyperhomocysteinemia and decreased methylation capacity, with neuropathology and aortic lipid deposition. Hum Mol Genet 2001;10(5):433-443.

64. Selhub J, Troen A, Rosenberg IH. B vitamins and the aging brain. Nutr Rev 2010:S112-S118.

65. Chan A, Tchantchou F, Graves V, Rozen R, Shea TB. Dietary and genetic compromise in folate availability reduces acetylcholine, cognitive performance and increases aggression: critical role of Sadenosyl methionine. J Nutr Health Aging 2008;12(4):252-261.

66. Linnebank M, Popp J, Smulders Y, et al. S-adenosylmethionine is decreased in the cerebrospinal fluid of patients with Alzheimer's disease. Neurodegener Dis 2010;7(6):373-378.

67. Sontag E, Hladik C, Montgomery L, et al. Downregulation of protein phosphatase $2 \mathrm{~A}$ carboxyl methylation and methyltransferase may contribute to Alzheimer disease pathogenesis. J Neuropathol Exp Neurol 2004;63(10):1080-1091.
68. Tian H, Lu Y, Liu J, et al. Leucine Carboxyl Methyltransferase Downregulation and Protein Phosphatase Methylesterase Upregulation Contribute Toward the Inhibition of Protein Phosphatase 2A by $\alpha$-Synuclein. Front Aging Neurosci 2018;10: 173.

69. Sontag E, Nunbhakdi-Craig V, Sontag JM, et al. Protein phosphatase 2A methyltransferase links homocysteine metabolism with tau and amyloid precursor protein regulation. J Neurosci 2007;27(11): 2751-2759.

70. Liu CD, Wang Q, Zong DK, et al. Knockdown of microRNA-195 contributes to protein phosphatase-2A inactivation in rats with chronic brain hypoperfusion. Neurobiol Aging 2016;45:76-87.

71. Martinez-Finley EJ, Gavin CE, Aschner M, Gunter TE. Manganese neurotoxicity and the role of reactive oxygen species. Free Radic Biol Med 2013;62:65-75.

72. Esteras N, Kundel F, Amodeo GF, et al. Insoluble tau aggregates induce neuronal death through modification of membrane ion conductance, activation of voltage-gated calcium channels and NADPH oxidase. FEBS J. 2020

73. Wang X, Tang S, Qin F, et al. Proteomics and phosphoproteomics study of LCMT1 overexpression and oxidative stress: overexpression of LCMT1 arrests HO-induced lose of cells viability. Redox Rep : communications in free radical research 2019;24(1):1-9.

74. Luka Z, Capdevila A, Mato JM, Wagner C. A glycine Nmethyltransferase knockout mouse model for humans with deficiency of this enzyme. Transgenic Res 2006;15(3):393-397.

75. Mingorance J, Alvarez L, Sánchez-Góngora E, Mato JM, Pajares MA. Site-directed mutagenesis of rat liver S-adenosylmethionine synthetase. Identification of a cysteine residue critical for the oligomeric state. Biochem J. 1996;315:761-766.

76. Pajares MA, Durán C, Corrales F, Pliego MM, Mato JM. Modulation of rat liver S-adenosylmethionine synthetase activity by glutathione. J Biol Chem 1992;267(25):17598-17605.

77. Kloypan C, Srisa-art M, Mutirangura A, Boonla C. LINE-1 hypomethylation induced by reactive oxygen species is mediated via depletion of S-adenosylmethionine. Cell Biochem Funct 2015;33(6):375-385.

78. V Venkataramani, TR Doeppner, D Willkommen, et al. Manganese causes neurotoxic iron accumulation via translational repression of amyloid precursor protein and H-Ferritin. J Neurochem 2018;147(6):831-848.

79. Foley TD, Melideo SL, Healey AE, Lucas EJ, Koval JA. Phenylarsine oxide binding reveals redox-active and potential regulatory vicinal thiols on the catalytic subunit of protein phosphatase 2A. Neurochem Res 2011;36(2):232-240.

80. Tang S, Lu C, Mo L, et al. Hydrogen peroxide redistributes the localization of protein phosphatase methylesterase 1. Life Sci 2018;213:166-173.

81. Stephenson AP, Schneider JA, Nelson BC, et al. Manganeseinduced oxidative DNA damage in neuronal SH-SY5Y cells: attenuation of thymine base lesions by glutathione and $\mathrm{N}$-acetylcysteine. Toxicol Lett 2013;218(3):299-307.

82. Reynolds E. Vitamin B12, folic acid, and the nervous system. Lancet Neurol 2006;5(11):949-960.

83. Choi SW, Friso S. Epigenetics: A New Bridge between Nutrition and Health. Advances in nutrition (Bethesda, Md). 2010;1(1):8-16

Publisher's Note Springer Nature remains neutral with regard to jurisdictional claims in published maps and institutional affiliations. 